\title{
Static versus Dynamic Deferred Acceptance in School Choice: Theory and Experiment*
}

\author{
Flip Klijn ${ }^{\dagger} \quad$ Joana Pais ${ }^{\ddagger} \quad$ Marc Vorsatz ${ }^{\S}$
}

September 18, 2018

\begin{abstract}
In the context of school choice, we experimentally study how behavior and outcomes are affected when, instead of submitting rankings in the student-proposing or school-proposing deferred acceptance (DA) mechanism, students make decisions dynamically, going through the steps of the underlying algorithms. Our main results show that, contrary to theory, (a) in the dynamic student-proposing DA mechanism, students propose to schools respecting the order of their true preferences slightly more often than in its static version while, (b) in the dynamic school-proposing DA mechanism, students react to proposals by always respecting the order and not accepting schools in the tail of their true preferences more often than in the corresponding static version. As a consequence, the dynamic mechanisms outperform their static counterparts in what stability and average payoffs are concerned. In the aggregate, the dynamic school-proposing DA mechanism is the best performing mechanism.
\end{abstract}

Keywords: dynamic school choice, deferred acceptance, stability, efficiency.

JEL-Numbers: C78, C91, C92, D78, I20.

*We are very grateful to two anonymous referees for their comments and suggestions that helped to improve the paper.

†Institute for Economic Analysis (CSIC) and Barcelona GSE, Campus UAB, 08193 Bellaterra (Barcelona), Spain; e-mail: flip.klijn@iae.csic.es. He gratefully acknowledges financial support from AGAUR-Generalitat de Catalunya (2014-SGR-1064 and 2017-SGR-1359), the Spanish Ministry of Economy and Competitiveness through Plan Estatal de Investigación Científica y Técnica y de Innovación 2013-2016 (ECO2014-59302-P and ECO201788130-P), and the Severo Ochoa Programme for Centres of Excellence in R\&D (SEV-2015-0563).

${ }^{\ddagger}$ ISEG-UL, Universidade de Lisboa; REM-Research in Economics and Management; UECE-Research Unit on Complexity and Economics; e-mail: jpais@iseg.ulisboa.pt. She gratefully acknowledges financial support from the Fundação para a Ciência e a Tecnologia under project reference no. PTDC/IIM-ECO/4546/2014.

$\S$ Departamento de Análisis Económico, Universidad Nacional de Educación a Distancia (UNED), Paseo Senda del Rey 11, 28040 Madrid, Spain; e-mail: mvorsatz@cee.uned.es. He gratefully acknowledges financial support from the Spanish Ministry of Economy and Competitiveness, through project ECO2015-65701-P. 


\section{Introduction}

\section{Motivation}

In school choice programs, parents can express preferences regarding the assignment of their children. Abdulkadiroğlu and Sönmez [1] show that prominent assignment procedures in the US had serious shortcomings (e.g., inefficiencies, manipulability) and employed matching theory to find a remedy. Under the student-proposing deferred acceptance (DA) mechanism suggested in Abdulkadiroğlu and Sönmez [1], students submit an ordered list of schools to a central authority that then employs a matching algorithm to determine the final allocation of students to schools. This mechanism induces therefore a "static" game in which the students' strategies (or rankings, i.e., ordered lists) may or may not correspond to their true preferences. Their seminal paper has triggered a considerable theoretical and a growing experimental literature.

Much of the experimental literature has concentrated on showing that under the static studentproposing DA mechanism subjects do not realize that it is in their best interest to report the ranking that coincides with their true preferences (truth-telling). The reported truth-telling rates are between $47 \%$ and $75 \%$ in Chen and Kesten [8], 64\% in Chen and Sönmez [11], between $44 \%$ and $65 \%$ in Klijn et al. [32], and between $67 \%$ and $82 \%$ in Pais and Pintér [35]. There is also a small literature that uses field data and makes this observation, e.g., Hassidim et al. [28] for the admission process in Israel for graduate studies in psychology and Rees--Jones [36] for the matching of medical students to residencies in the US.

The school-proposing version of the DA mechanism uses the same algorithm, but with roles reversed (schools make proposals and students receive offers). This static school-proposing DA mechanism has not been advocated in the school choice theory, since it is well-known that for this mechanism students have in general incentives to misrepresent their preferences, but it is nevertheless used in practice. ${ }^{1}$ Featherstone and Mayefsky [17] however find in a laboratory experiment that subjects reveal their true preferences too often (i.e., manipulate not often enough). Castillo and Dianat [6] experimentally study a restricted version of the same problem in which a particular form of "truncation" strategy, whereby a student may remove a tail of least preferred schools from her true preference list, is always a best response. They find that truncation is not sensitive to considerations of profitability, but it is sensitive to the risk of remaining unmatched.

The objective of our paper is to analyze by means of a laboratory experiment whether behavior is affected and better outcomes are obtained if, instead of submitting rankings to a central authority, subjects make decisions dynamically. Dynamic implementations of matching algorithms are used in college admissions in Brazil (Bó and Hakimov [3]) and in Inner Mongolia (Chen and Kesten [9] and Gong and Liang [22]), and in school choice in the Wake County Public School System (Dur et al. [15]). A dynamic version of the deferred acceptance procedure was used for some time in the entry-level job market for clinical psychologists (Roth and Xing [40]). Inspired by these markets,

\footnotetext{
${ }^{1}$ Examples of matching markets where variants of the school-proposing DA mechanism are used are the assignment of teachers to schools and students to secondary schools in France (Terrier [42] and Hiller and Tercieux $[29],[30]$ ), students to secondary schools in Finland (Salonen [41]), and university admission in Germany (Braun et al. [4] and Westkamp [43]).
} 
Chen and Pereyra [10] theoretically study a dynamic matching mechanism where at each stage students propose to a school and receive information about the tentative matching. Contrary to a dynamic version of the student-proposing DA, students are allowed to revise their proposals and the number of stages is bounded. Chen and Pereyra [10] conclude that this dynamic mechanism can generate a higher social surplus than the (standard) student-proposing DA.

In the dynamic mechanisms we test in this paper, subjects go through the steps of an algorithm, either the student-proposing or the school-proposing DA. In the former case, subjects are prompted to send a new application upon receiving a rejection and, in the latter case, to immediately react to each offer received. This step-by-step approach may help subjects in an experiment to understand better how the underlying algorithm works. More importantly, by receiving feedback about their current status (direct experience), subjects have the possibility to use the information received, adapt behavior, and revise their strategies while the assignment process proceeds.

\section{Theory}

We first provide a theoretical analysis of the extensive-form games induced by the dynamic student-proposing and the dynamic school-proposing DA mechanisms when each school has exactly one seat. These results help to understand the incentives in the dynamic games and serve as a null hypothesis for our experiment (when compared with the incentives in the static implementations).

For the student-proposing DA mechanism we find that every Nash equilibrium outcome of the static game is also a Nash equilibrium outcome of the dynamic game. Since the set of stable matchings is a subset of the set of Nash equilibrium outcomes of the static game (see, e.g., Haeringer and Klijn [26]), every stable matching can also be sustained as an equilibrium outcome of the dynamic game. However, while proposers have incentives to submit lists that coincide with their true preferences in the static game, we show by means of examples that for all markets we consider throughout the experiment, the strategy of "going down the list with respect to the true preferences," i.e., always applying to the best school that a student has not applied to yet, is not a weakly dominant strategy in the dynamic game, even though it remains a best reply when the other players also act according to a list (not necessarily corresponding to their true preferences).

We show for the school-proposing DA mechanism that the set of Nash equilibrium outcomes is equal to the set of stable matchings. Then, since the set of Nash equilibrium outcomes in the static game is equal to set of stable matchings whenever each school has exactly one seat (Gale and Sotomayor [21], Roth [38]), we can conclude that the sets of Nash equilibrium outcomes coincide for both implementations in our setting. Moreover, it is well-known that the static mechanism is manipulable and that the set of truncation strategies is strategically exhaustive in the sense that for each strategy a student may use, the induced match can be replicated or improved upon by some truncation of her true preferences (see, e.g., Jaramillo et al. [31] and Roth and Vande Vate [39]). Nevertheless, in the dynamic game we can construct strategy profiles for

the markets we test experimentally so that for all truncations of the true preferences, going down 
the list with respect to the truncation is not a best reply. So, a wider range of behavior can again be supported for the dynamic implementation.

\section{Laboratory experiments}

Our laboratory experiment is designed to analyze the effects of a dynamic implementation of the DA mechanism. We consider four problems with four students and four schools with one seat each that differ in the degree of heterogeneity of preferences of students and priorities of schools. In consistence with the theoretical analysis, subjects assume the role of students, schools are passive and simply follow their priorities. In order to account for the possibility of learning, each problem is played six times throughout an experimental session. Our treatment conditions correspond to the different matching mechanisms. The two benchmark treatments are the static student-proposing DA mechanism (treatment CI) and the static school-proposing DA mechanism (treatment CS). These treatments are compared with their dynamic counterparts, the dynamic student-proposing DA mechanism (treatment DI) and the dynamic school-proposing DA mechanism (treatment DS).

The experimental results show that subjects are often not truthful as proposers, but they respect the true order of their preferences slightly more often in treatment DI than in treatment CI. As a consequence, the dynamic student-proposing DA mechanism weakly outperforms the static implementation in terms of stability and efficiency, that is, the frequency with which a stable matching is reached and the average payoff are never lower and, in some problems, significantly higher for the dynamic mechanism. On the other hand, for the school-proposing DA mechanisms, it turns out that subjects react to proposals by respecting their true order and by not accepting schools in the tail of their true preferences significantly more often in the dynamic than in the static implementation. This leads to the dynamic school-proposing DA mechanism weakly outperforming the static counterpart in terms of stability and efficiency. In fact, the dynamic school-proposing DA mechanism weakly outperforms even the two student-proposing DA mechanisms in these two dimensions.

Several recent experimental papers look into dynamic implementations of deferred acceptance. Haruvy and Ünver [27] study repeated interactions between the two sides of the market. They do so under two information levels on others' preferences and, contrary to our experiment, allow proposers to repeat offers. Haruvy and Ünver [27] find that acting according to the true preferences is a good predictor of proposers' behavior, particularly under low information, and that the proposer-optimal stable matching is reached in most cases. Our paper is most closely related to the recent studies by Bó and Hakimov [2], Castillo and Dianat [7], Dur et al. [14], and Echenique et al. [16] who report arrays of laboratory experiments where subjects go through the steps of the DA algorithm. ${ }^{2}$ One important difference with respect to our paper is that in Castillo and Dianat [7] and in Echenique et al. [16] both sides of the market are active, whereas we take schools to be passive. ${ }^{3}$ Consequently, in our setting subjects do not have to

\footnotetext{
${ }^{2}$ Gong and Liang [22] also study dynamic assignment mechanisms in the lab, but instead of going through the steps of an algorithm, they provide feedback and allow subjects to revise their choices.

${ }^{3}$ Castillo and Dianat [7] is an extension of Echenique et al. [16] to test the impact of different levels of information about others' preferences on behavior and outcomes.
} 
form beliefs about the behavior of the other side of the market, a simplification that could help in making better decisions. This is true in what receivers' behavior is concerned, as receivers fail to truncate in Castillo and Dianat [7] and in Echenique et al. [16], while often reverting to this strategy in our treatment DS. In fact, and as mentioned above, receivers act according to a truncation significantly more often in the dynamic school-proposing DA than in its static version, a comparison that is absent in the other two papers. Moreover, in Echenique et al. [16] a stable matching (mainly the receiver-optimal one) is reached in about $48 \%$ of the cases, whereas in Castillo and Dianat [7] a stable matching is obtained in $55 \%$ of the cases in which subjects hold complete information. We obtain higher frequencies (between $64 \%$ in treatment CI and $90 \%$ in treatment DS). Bó and Hakimov [2] compare the static and the dynamic versions of the student-proposing DA mechanism and show that the dynamic implementation leads more often to a stable outcome than the static one. We find significant differences for some but not all problems we study, which highlights that design details matter. Bó and Hakimov [2] generate each round randomly a new market following the procedures of Chen and Sönmez [11], while we truly repeat each of our four markets six times. Also, in Bó and Hakimov [2] markets are larger and almost always have a unique stable matching, whereas the number of stable matchings ranges from 1 to 4 in our markets. But, most importantly, Bó and Hakimov [2] work in an incomplete information setting as subjects have uncertainty regarding priorities. In our experiment, on the other hand, subjects have complete information. Finally, Dur et al. [14] compare implementations of the Boston Mechanism (BM) with the student-proposing DA, where agents sequentially submit ordered lists of schools knowing previously submitted strategies (and the algorithm is run only once, when all students have submitted their lists). They show that under a natural equilibrium refinement, this sequential implementation is more efficient under BM than under DA. More specifically, for any problem, any equilibrium outcome under BM (weakly) Pareto dominates the student-optimal stable matching. Field data and their laboratory experiment provide suggestive evidence of these efficiency gains.

\section{Organization}

We proceed as follows. In Section 2, we introduce the experimental design and procedures. Section 3 contains the theoretical analysis. In Section 4, we present and discuss our experimental results. In Section 5, we conclude. Most proofs are relegated to Appendix A. Additional figures and tables are displayed in Appendix B. The detailed experimental instructions are relegated to Appendix C.

\section{Laboratory experiment}

Our experiment is designed to analyze the differences between a static and a dynamic implementation of the DA mechanism in school choice problems. The students $i_{1}, i_{2}, i_{3}$, and $i_{4}$ seek to obtain a seat at the schools $s_{1}, s_{2}, s_{3}$, and $s_{4}$. Each school offers exactly one seat. The preferences of the students and the priorities of the schools in the four problems are depicted in Table 1 . The 
information in Table 1 is common knowledge.

\begin{tabular}{|c|c|c|c|c|c|c|c|c|}
\hline \multirow[t]{2}{*}{ Problem 1} & \multicolumn{4}{|c|}{ Preferences } & \multicolumn{4}{|c|}{ Priorities } \\
\hline & $i_{1}$ & $i_{2}$ & $i_{3}$ & $i_{4}$ & $s_{1}$ & $s_{2}$ & $s_{3}$ & $s_{4}$ \\
\hline Best match & $s_{1}$ & $s_{2}$ & $s_{3}$ & $s_{4}$ & $i_{2}$ & $i_{3}$ & $i_{4}$ & $i_{1}$ \\
\hline Second best & $s_{2}$ & $s_{3}$ & $s_{4}$ & $s_{1}$ & $i_{3}$ & $i_{4}$ & $i_{1}$ & $i_{2}$ \\
\hline Third best & $s_{3}$ & $s_{4}$ & $s_{1}$ & $s_{2}$ & $i_{4}$ & $i_{1}$ & $i_{2}$ & $i_{3}$ \\
\hline Worst match & $s_{4}$ & $s_{1}$ & $s_{2}$ & $s_{3}$ & $i_{1}$ & $i_{2}$ & $i_{3}$ & $i_{4}$ \\
\hline \multirow[t]{2}{*}{ Problem 3} & \multicolumn{4}{|c|}{ Preferences } & \multicolumn{4}{|c|}{ Priorities } \\
\hline & $i_{1}$ & $i_{2}$ & $i_{3}$ & $i_{4}$ & $s_{1}$ & $s_{2}$ & $s_{3}$ & $s_{4}$ \\
\hline Best match & $s_{2}$ & $s_{2}$ & $s_{4}$ & $s_{4}$ & $i_{2}$ & $i_{4}$ & $i_{4}$ & $i_{2}$ \\
\hline Second best & $s_{1}$ & $s_{3}$ & $s_{3}$ & $s_{1}$ & $i_{3}$ & $i_{3}$ & $i_{1}$ & $i_{1}$ \\
\hline Third best & $s_{3}$ & $s_{4}$ & $s_{1}$ & $s_{2}$ & $i_{4}$ & $i_{1}$ & $i_{2}$ & $i_{3}$ \\
\hline Worst match & $s_{4}$ & $s_{1}$ & $s_{2}$ & $s_{3}$ & $i_{1}$ & $i_{2}$ & $i_{3}$ & $i_{4}$ \\
\hline
\end{tabular}

\begin{tabular}{|c|c|c|c|c|c|c|c|c|}
\hline \multirow[t]{2}{*}{ Problem 2} & \multicolumn{4}{|c|}{ Preferences } & \multicolumn{4}{|c|}{ Priorities } \\
\hline & $i_{1}$ & $i_{2}$ & $i_{3}$ & $i_{4}$ & $s_{1}$ & $s_{2}$ & $s_{3}$ & $s_{4}$ \\
\hline Best match & $s_{2}$ & $s_{3}$ & $s_{4}$ & $s_{1}$ & $i_{2}$ & $i_{2}$ & $i_{4}$ & $i_{4}$ \\
\hline Second best & $s_{3}$ & $s_{4}$ & $s_{1}$ & $s_{2}$ & $i_{1}$ & $i_{3}$ & $i_{3}$ & $i_{1}$ \\
\hline Third best & $s_{4}$ & $s_{1}$ & $s_{2}$ & $s_{3}$ & $i_{3}$ & $i_{4}$ & $i_{1}$ & $i_{2}$ \\
\hline Worst match & $s_{1}$ & $s_{2}$ & $s_{3}$ & $s_{4}$ & $i_{4}$ & $i_{1}$ & $i_{2}$ & $i_{3}$ \\
\hline \multirow[t]{2}{*}{ Problem 4} & \multicolumn{4}{|c|}{ Preferences } & \multicolumn{4}{|c|}{ Priorities } \\
\hline & $i_{1}$ & $i_{2}$ & $i_{3}$ & $i_{4}$ & $s_{1}$ & $s_{2}$ & $s_{3}$ & $s_{4}$ \\
\hline Best match & $s_{1}$ & $s_{1}$ & $s_{1}$ & $s_{1}$ & $i_{1}$ & $i_{1}$ & $i_{1}$ & $i_{1}$ \\
\hline Second best & $s_{2}$ & $s_{2}$ & $s_{2}$ & $s_{2}$ & $i_{4}$ & $i_{2}$ & $i_{2}$ & $i_{2}$ \\
\hline Third best & $s_{3}$ & $s_{3}$ & $s_{3}$ & $s_{3}$ & $i_{3}$ & $i_{4}$ & $i_{3}$ & $i_{3}$ \\
\hline Worst match & $s_{4}$ & $s_{4}$ & $s_{4}$ & $s_{4}$ & $i_{2}$ & $i_{3}$ & $i_{4}$ & $i_{4}$ \\
\hline
\end{tabular}

Table 1: Preferences of students over schools and priorities of schools over students.

We selected the four problems in such a way that a wide variety of situations is covered. In Problem 1, each student is ranked last in her most preferred, third in her second most preferred, second in her third most preferred, and first in her least preferred school. This problem thus resembles a situation where preferences (and priorities) are heterogeneous and the tension between the two sides of the market is maximal. The opposite case, homogeneous preferences (and priorities in the relevant parts) and no conflict between the two sides of the market, corresponds to Problem 4. Problems 2 and 3 are intermediate situations.

During the experiment, subjects assume the role of students. Schools are not strategic players. Given the information in Table 1, the subjects' task in the static mechanisms is to submit an ordered list (ranking) of schools (not necessarily the true preferences) to be used by a central clearinghouse to assign students to schools. Subjects are free to submit a list of less than four schools. So, for example, a subject is allowed to submit the ranking $s_{3}, s_{1}$. We consider two static mechanisms, the "student-proposing deferred acceptance" (treatment CI) and the "school-proposing deferred acceptance" (treatment CS) mechanisms. For the particular school choice settings at hand, the two mechanisms are as follows:

\section{Static MEChanisms (CI AND CS)}

\section{Step 1 .}

(CI) Each student sends an application to the school she ranked first. If a student did not list any school, then she will not send any application.

Each school temporarily accepts the applicant with the highest priority and rejects all other applicants. 
(CS) Each school offers a seat to its highest priority student.

Each student temporarily accepts the school she likes best according to her submitted ranking and rejects all other offers.

\section{Step 2 .}

(CI) Each student who got rejected in the previous step sends an application to her next highest ranked school. If there is no such school, then the student will not send any application.

Among the previously retained (if any) and new applications, each school temporarily accepts the applicant with the highest priority and rejects all other applicants.

(CS) Each school that got rejected in the previous step makes an offer to its next highest priority student.

Among the previously retained (if any) and new offers, each student temporarily accepts the school she likes best according to her submitted ranking and rejects all other offers.

\section{Steps 3, 4, . .}

(CI) Step 2 is repeated until no more applications are rejected. Each student is assigned to the school that holds her application. If a student got rejected by all schools she ranked, she is left unmatched.

(CS) Step 2 is repeated until no more offers are rejected. Each student is assigned to the school she holds the offer from. If a student does not hold an offer, she is left unmatched.

In our dynamic implementations students do not submit a ranking to the central clearinghouse. Instead, they go through each step of the corresponding static algorithm. So, while the static mechanisms induce a simultaneous move game, in the dynamic implementation subjects face a sequential move game. The descriptions of the dynamic versions of the student-proposing DA mechanism (treatment DI) and the school-proposing DA mechanism (treatment DS) are as follows:

\section{DYNAMIC MECHANISMS (DI AND DS)}

\section{Phase 1.}

(DI) Each student is asked to send an application that either contains the name of a school or is left empty. If a student decides to send an empty application, she will not be able to send future applications.

Each school temporarily accepts the applicant with the highest priority and rejects all other applicants.

Each student is informed about the status of the application, that is, if it is retained or whether it got rejected. If a student sent an empty application, she is informed that she did not obtain a seat. 
(DS) Each school offers a seat to its highest priority student.

Each student decides to retain at most one of the offers received.

\section{Phase 2.}

(DI) The students who sent an application to a school in the previous phase and got rejected are asked to send a new application (that consists of a school the student has not yet applied to or is left empty). If a student decides to send an empty application, she will not be able to send future applications.

Among the previously retained (if any) and new applications, each school temporarily accepts the applicant with the highest priority and rejects all other applicants.

Each student who sent a new application to a school and each student whose application was retained in the previous phase is informed about the status of the application, that is, if it is retained or whether it got rejected. Each student who sent an empty application is informed that she did not obtain a seat.

(DS) Whenever a school's offer is rejected by a student, the school makes an offer to its next highest priority student.

Each student who received at least one new offer can retain at most one offer (among the new offers and the retained one, if it exists).

\section{Phases 3, 4, . .}

(DI) Phase 2 is repeated until each student decides not to send any further application, has been rejected by all schools, or has her application retained by a school. Each student is assigned to the school that holds her application. If a student got rejected by all schools or sent an empty application, she is left unmatched.

(DS) Phase 2 is repeated until no more offers are rejected. Each student is assigned to the school she holds the offer from. If a student does not hold an offer, she is left unmatched.

The experiment was programmed within the z-Tree toolbox provided by Fischbacher [19] and carried out at Lineex (www.lineex.es) hosted at the University of Valencia. In total, 192 undergraduates from various disciplines participated in the experiment. We ran one session with 48 subjects per treatment. At the beginning of the session, subjects were anonymously matched into groups of four. Within each group, one subject was assigned the role of student $i_{1}$, another subject the role of $i_{2}$, and so forth. Groups and roles did not change over the course of the experiment. Participants were told that the experiment would take a total of 24 rounds and that preferences and priorities would change each six rounds. Before the first round, subjects went individually over an illustrative example in order to get used to the matching procedure. Afterwards, we implemented a trial period that was not taken into account for payment. This helped subjects to get familiar with the computer software. At the beginning of each round, the computer screen presented the preferences of all group members and the priorities of the four schools. Subjects took then their 
respective decisions. At the end of each round, subjects got to know the outcome of the matching process. In treatments DI and DS, due to the sequential nature of the game, subjects knew the status of their application at each point of the process. No explicit information about the behavior of the other group members was provided. At the end of the experiment, one round was randomly selected for payment. Subjects received 24, 20, 16, and 12 experimental currency units (ECU) if they ended up in their most, second most, third most, and least preferred school. They got 7 ECU if they remained unmatched. Each ECU was worth 1 Euro. The experimental instructions are available in Appendix C. ${ }^{4}$

\section{Theory}

In this section we start with the definition of a school choice problem (Section 3.1) and then provide a broad description of the extensive-form games induced by the two dynamic mechanisms, DI and DS (Section 3.2). Since the two mechanisms differ in very important aspects, we then focus on each game in turn (Sections 3.3 and 3.4).

\subsection{School choice problem}

A school choice problem is a five-tuple $\left\langle I, S, q, P_{S}, P_{I}\right\rangle$ where

- $I$ is a finite set of students (individuals).

- $S$ is a finite set of schools.

- $q=\left(q_{s}\right)_{s \in S}$, with $q_{s}=1$ for each $s \in S$, where $q_{s}$ is the number of seats at $s \in S$.

- $P_{S}=\left(P_{s}\right)_{s \in S}$ is a profile of strict priority relations for schools, where $P_{s}$ is a complete, irreflexive, and transitive binary relation over $I \cup\{s\}$ for each $s \in S$.

- $P_{I}=\left(P_{i}\right)_{i \in I}$ is a profile of strict preference relations for students, where $P_{i}$ is a complete, irreflexive, and transitive binary relation over $S \cup\{i\}$ for each $i \in I$.

For each $s \in S$ and each $i \in I$, we say that $s$ finds $i$ acceptable if $i P_{s} s$ and we say that $i$ finds $s$ acceptable if $s P_{i} i$. In the problems we consider, a matching of students to schools is a function $\mu: I \cup S \rightarrow I \cup S$ such that for all $i \in I$ and $s \in S$ :

- $\mu(i) \in S \cup\{i\}$

- $\mu(s) \in I \cup\{s\}$, and

- $s=\mu(i)$ if and only if $i=\mu(s)$,

\footnotetext{
${ }^{4}$ The $\mathrm{z}$-Tree programs, data files, and $\mathrm{R}$ programs are available from the authors upon request.
} 
where the notation $v=\mu(v)$ means that agent $v$ is unmatched under $\mu$ and $v^{\prime}=\mu(v)$ denotes that agent $v^{\prime}$ is agent $v$ 's partner under matching $\mu$. Let $\mathcal{M}$ denote the set of matchings.

A matching $\mu$ is individually rational if $\mu(v) P_{v} v$ for all $v \in V$. Moreover, we say that a pair $(i, s)$ blocks the matching $\mu$ if $s P_{i} \mu(i)$ and $i P_{s} \mu(s)$. Since in the problems we consider each school finds each student acceptable and likewise each student finds each school acceptable, each matching in $\mathcal{M}$ is individually rational. Therefore, we say that a matching is stable if there is no pair that blocks it. We denote by $S(P)$ the set of all stable matchings. The set of stable matchings is always non-empty (Gale and Shapley [20]). Moreover, for each problem, there is a student-optimal stable matching that is weakly preferred by all students to any other stable matching in $S(P)$; similarly, there is a school-optimal stable matching that is weakly preferred by all schools to any other stable matching in $S(P)$ (Gale and Shapley [20]).

\subsection{General description of the extensive-form games}

Given the definition of a school choice problem, we are now in a position to describe the extensiveform games agents play when confronted with the dynamic mechanisms, DI and DS. The extensiveform games are composed of the following elements:

- A finite set of players $I$, which corresponds to the set of students.

- A set of actions $A_{i}=S \cup\{i\}$ for each student $i$. In DI, this set includes all possible proposals that might potentially be made by $i$ at some point in the game, including the "no school" option (modelled as proposing to oneself). In DS, choosing $s$ means to hold the proposal from school $s$ and to reject every other proposal that might have been received or held from a previous step, and to choose $i$ means rejecting all proposals. Let $A \equiv \cup_{i \in I} A_{i}$.

- A set of nodes or histories $X$, where

- the initial node or empty history $x_{0}$ is an element of $X$,

- each $x \in X \backslash\left\{x_{0}\right\}$ takes the form $x=\left(a_{1}, a_{2}, \ldots, a_{k}\right)$ for some finitely many actions $a_{i} \in A$, and

- if $\left(a_{1}, a_{2}, \ldots, a_{k}\right) \in X \backslash\left\{x_{0}\right\}$ for some $k>1$, then $\left(a_{1}, a_{2}, \ldots, a_{k-1}\right) \in X \backslash\left\{x_{0}\right\}$.

When $x=\left(x^{\prime}, a_{1}, \ldots, a_{k}\right), k \geq 1$, we say that $x^{\prime}$ is a predecessor of $x$.

Let $A(x) \equiv\{a \in A:(x, a) \in X\}$ denote the set of actions available to a student whose turn it is to move after history $x \in X$. Clearly, we have $A(x) \subseteq S \cup\{i\}$ for all $x \in X$ where it is student $i$ 's turn to move.

- A set of end nodes $E \equiv\{x \in X:(x, a) \notin X$ for all $a \in A\}$.

- A function $\iota: X \backslash E \rightarrow I$ that indicates whose turn it is to move at each decision node in $X \backslash E$.

Let $X_{i} \equiv\{x \in X \backslash E: \iota(x)=i\}$ denote the set of decision nodes belonging to student $i$. 
- A partition $\Im$ of the set of decision nodes $X \backslash E$, such that if $x$ and $x^{\prime}$ are in the same element of the partition, then $(i) \iota(x)=\iota\left(x^{\prime}\right)$, and $(i i) A(x)=A\left(x^{\prime}\right)$. The information available to student $\iota(x)$ when, after history $x$, it is her turn to move is described by $\Im(x)$, the element of the partition $\Im$ that contains $x$.

Let $\Im_{i} \equiv\{\Im(x): x \in X \backslash E$ with $\iota(x)=i\}$ denote the set of information sets belonging to student $i$.

- An outcome function $g: E \rightarrow \mathcal{M}$ that associates each terminal history with a matching.

- For each student $i \in I$, a preference ordering $P_{i}$ of schools.

We are now in a position to give a definition of a strategy in this extensive-form game.

Definition 1: A (pure) strategy for student $i$ in this extensive-form game is a function $\sigma_{i}: \Im_{i} \rightarrow A$ such that $\sigma_{i}(\Im(x)) \in A(x)$ for all $x \in X_{i}$.

Finally, even though we have provided a general description of the games induced by both DI and DS, they are clearly different mechanisms. For example, the set of actions available at a node $x$ and the set of students allowed to move depend on the particular rules of the games induced by each mechanism. In Sections 3.3 and 3.4, we focus on each of these games in turn.

\subsection{Game induced by DI}

Two rules of DI, which we describe next, are of special importance.

Rule 1: In DI students cannot pass turns, i.e., if a student sends an application to "no school" at some step, she is not allowed to send applications at future steps.

Rule 2: In DI a student cannot send an application to a school more than once.

Whenever a student makes a new proposal it is because she was rejected and by Rule 2 she makes a new proposal to "no school" or to a school she has not proposed to before. Moreover, by Rule 1, after proposing to "no school" the student can no longer make proposals.

Therefore, in what the strategy of a student is concerned, her observed decisions induce an ordered list. Formally, consider student $i$, a profile of strategies $\sigma=\left(\sigma_{i}, \sigma_{-i}\right)$ leading to $x \in E$, and let $x^{1}, \ldots, x^{k}, k \geq 1$, denote all the predecessors of $x$ where student $i$ proposes. Without loss of generality, let $x^{j}$ be a predecessor of $x^{j+1}, j=1, \ldots, k-1$.

Definition 2: In the game induced by DI, the ordered list revealed by student $i$ in the play of $\sigma$ is $Q_{i}^{\sigma}=\sigma_{i}\left(\Im\left(x^{1}\right)\right), \ldots, \sigma_{i}\left(\Im\left(x^{k}\right)\right)$, if $\sigma_{i}\left(\Im\left(x^{k}\right)\right)=i$, and $Q_{i}^{\sigma}=\sigma_{i}\left(\Im\left(x^{1}\right)\right), \ldots, \sigma_{i}\left(\Im\left(x^{k}\right)\right), i$, otherwise.

Note that no two elements of this list are the same, so that this is a strict partial order of schools, having $i$ as a last element. In fact, by Rule 1, $i$ can only be the last element of this list and, by Rule 2, no school can appear twice. 
Claim 1: The outcome of the game induced by DI when students use $\sigma$ coincides with the outcome of the game induced by CI when students submit the ordered lists revealed in the play of $\sigma$.

Proof. Immediate from the definition of ordered lists revealed in the play of a strategy profile and from the fact that DI emulates CI.

Still, the information revealed in the play of a strategy leading to a particular terminal node may only provide a partial order of schools, so that several (complete) orders may be compatible with it. Moreover, the strategy space is clearly much wider in DI than in CI, so that one strategy in DI may lead to different revealed ordered lists.

A class of strategies that deserves attention for its simplicity is the class of preference strategies. A student using a preference strategy always proposes to the best ranked school that she has not proposed to yet according to some preference ordering. Formally, consider student $i$, let $Q_{i}$ be an ordered list of preferences over the elements of $S \cup\{i\}$, and for any $S^{\prime} \subseteq S \cup\{i\}$ with $i \in S^{\prime}$ let $\max _{Q_{i}}\left(S^{\prime}\right)$ be the best ranked element according to $Q_{i}$ in the set $S^{\prime}$.

Definition 3: In the game induced by DI, $\sigma_{i}$ is a preference strategy if there exists an ordered list $Q_{i}$ for student $i$ such that for all $x \in X_{i}, \sigma_{i}(\Im(x))=\max _{Q_{i}}(A(x))$. In this case, we say that $\sigma_{i}$ is the preference strategy consistent with $Q_{i}$.

Remark 1: When student $i$ uses the preference strategy consistent with $Q_{i}$, the ordered list that is revealed (at least partially) in any play of the game is $Q_{i}$.

Claim 2: Every Nash equilibrium outcome in the game induced by CI is a Nash equilibrium outcome in the game induced by DI.

Proof. See Appendix A.

Haeringer and Klijn [26] show that every stable and possibly some unstable matchings (when the priority structure exhibits so-called Ergin cycles) can be sustained in Nash equilibrium in the game induced by CI. It therefore follows from Claim 2 that the same result holds in the game induced by DI.

Finally, whereas submitting the true preferences to the static mechanism is a weakly dominant strategy (Dubins and Freedman [13] and Roth [37]), the preference strategy consistent with the true preferences is not weakly dominant in the game induced by DI. In fact, when the other players use strategies that are not preference strategies, deviating from the preference strategy consistent with the true preferences may trigger the revelation of other, more favorable ordered lists by the other students and lead to a better outcome.

Claim 3: In the game induced by DI, the preference strategy consistent with the true preferences is in general not a weakly dominant strategy.

Proof. See Appendix A. 
Additionally, in the game induced by DI, due to the lack of information on others' moves (as the game proceeds a student remembers her own proposals and schools' reactions to these proposals), there are very few proper subgames, and those that do exist appear only at the end of the tree. It follows that the concept of subgame perfect Nash equilibrium does not allow us to refine the set of predicted outcomes either and our best prediction for the obtained matchings is a superset of the Nash equilibrium outcomes in the game induced by CI.

\subsection{Game induced by DS}

It is a well-known fact that the set of Nash equilibrium outcomes of the game induced by CS when each school has one seat coincides with the set of stable matchings (Gale and Sotomayor [21], Roth [38]). In the following claim, we show that the set of Nash equilibrium outcomes in the game induced by DS coincides with the set of stable matchings and, therefore, with the set of Nash equilibrium outcomes in the game induced by CS.

Claim 4: The set of Nash equilibrium outcomes in the game induced by DS coincides with the set of stable matchings.

Proof. See Appendix A.

The following rule of DS is of special importance.

Rule 3: In DS once a student holds the proposal of a school, she can only reject it upon receiving and holding another school's proposal.

Since in the game induced by DS a student can receive a proposal from a school at most once, we can guarantee that the decisions made by a student along the path of a play reveal a strict partial order of schools. Moreover, each school whose proposal is held by a student is revealed to be better than being unmatched in which case, by Rule 3, being unmatched cannot be revealed preferred to being assigned to that school. Therefore, the rules of the game guarantee that transitivity can never be violated. Formally, consider student $i$, a profile of strategies $\sigma=\left(\sigma_{i}, \sigma_{-i}\right)$ leading to $x \in E$, and let $S^{\prime}$ be the set of schools held by student $i$ in the course of play of $\sigma$.

Definition 4: The ordered list that is revealed by student $i$ in the play of $\sigma$ is $Q_{i}^{\sigma}=i$, when $S^{\prime}=\emptyset$, and is $Q_{i}^{\sigma}=s_{k}^{\prime}, \ldots, s_{1}^{\prime}, i$, where $s_{j}^{\prime} \in S^{\prime}, j=1, \ldots, k$, is the $j^{\text {th }}$ school whose proposal is held by $i$ in the course of play of $\sigma$.

Claim 5: The outcome of the game induced by DS when students use $\sigma$ coincides with the outcome of the game induced by CS when students submit the ordered lists revealed in the play of $\sigma$.

Proof. Immediate from the definition of ordered lists revealed in the play of a strategy profile and from the fact that DS emulates CS. 
As it happens in the game induced by DI, the class of preference strategies is of particular interest. A student using a preference strategy always holds the proposal from the best ranked school in some ordered list of schools among those schools that propose at some step and the school whose proposal may be held from a previous step (if any). Formally, consider student $i$ and let $Q_{i}$ be an ordered list over the elements of $S \cup\{i\}$.

Definition 5: In the game induced by DS, $\sigma_{i}$ is a preference strategy for student $i$ if there exists an ordered list $Q_{i}$ such that for all $x \in X_{i}, \sigma_{i}(\Im(x))=\max _{Q_{i}}(A(x))$. In this case, we say that $\sigma_{i}$ is the preference strategy consistent with $Q_{i}$.

Preference strategies are a natural form of behavior and Claim 6 refers to a particular subclass, the subclass of preference strategies consistent with a truncation of the true preferences.

In the game induced by CS, a student employs a truncation strategy if she removes a (possibly empty) tail of least preferred schools from the true preference list. For example, if the true ranking is $s_{2}, s_{3}, s_{4}, s_{1}$, then $s_{2}, s_{3}$ and $s_{2}, s_{3}, s_{4}, s_{1}$ (the true ranking) are truncation strategies, but $s_{2}, s_{4}$ is not (if $s_{3}$ is removed, then $s_{4}$ should also be removed). In the game induced by CS, truncations may play an important role given that, for each strategy a student may use, the induced match can be replicated or improved upon by some truncation of the true preferences of the student (Roth and Vande Vate [39]). ${ }^{5}$ This fact, together with Claim 5 imply that, in the game induced by DS, given a profile of preference strategies for the students other than $i$, there is a best reply for $i$ that consists of a preference strategy consistent with a truncation of the true preferences.

Nevertheless, for some profiles of strategies of the other players (including strategies other than preference strategies), there is no strategy consistent with a truncation of the true preferences that can be a best reply in DS. Therefore, in contrast with what happens in CS, when choosing a best reply, an agent cannot restrict attention to the class of preference strategies that are consistent with some truncation of her true preferences.

Claim 6: Consider the game induced by DS. Given a strategy-profile of all but one student $i$, there need not be a best reply of student $i$ that is a preference strategy consistent with a truncation of her true preferences.

Proof. See Appendix A.

Finally, we can also show that, as it happens in DI, given the low level of information on other players' chosen strategies, the game induced by DS has very few subgames and subgame perfect Nash equilibrium does not give us sharper predictions on the possible outcomes. Therefore, in the game induced by DS, the set of subgame perfect Nash equilibrium outcomes coincides with the set of Nash equilibrium outcomes.

\footnotetext{
${ }^{5}$ In particular, this result on the "strategic exhaustiveness" of the class of truncation strategies (Theorem 2 in Roth and Vande Vate [39]) crucially depends on the fact that the true preferences constitute a (particular, degenerate) truncation strategy.
} 


\section{Experimental results}

We now present the results of our experiment. First, we analyze the behavior of the subjects (Section 4.1). Afterwards, we show how behavior affects stability (Section 4.2) and welfare (Section 4.3).

\subsection{Behavior}

\section{Student-proposing DA mechanisms}

Dubins and Freedman [13] and Roth [37] show that subjects have incentives to reveal preferences truthfully in treatment CI. Nevertheless, it is a well-documented finding in the experimental literature that agents do not always realize that it is in their best interest to reveal their true preferences when confronted with centralized strategy-proof mechanisms. ${ }^{6}$ We analyze whether a dynamic implementation of the student-proposing DA algorithm where, as the algorithm proceeds, subjects receive feedback about their previous applications and corresponding decisions, mitigates or aggravates this problem.

Given some student's true preferences, we write $s^{k}$ for the school that is ranked in $k$-th position (from best to worst), i.e., the $k$-th best school. For example, if the student's true ranking is $s_{2}, s_{3}, s_{4}, s_{1}$, then $s^{4}=s_{1}$.

Consider the mechanism CI. Haeringer and Klijn [26] show that a strategy that does not rank all schools is weakly dominated. Now suppose a student ranks all schools. Haeringer and Klijn [26] show that a strategy that for some $i<j$ ranks school $s^{j}$ somewhere above/before school $s^{i}$ is weakly dominated by a strategy that is obtained by switching $s^{j}$ and $s^{i}$ back in the (true) relative order. Therefore, the number of such switches can be employed to measure a subject's degree of (ir)rationality. For example, if the true ranking is $s_{2}, s_{3}, s_{4}, s_{1}$ and a subject reports in treatment CI the list $s_{4}, s_{2}$, she switches schools $s^{1}$ and $s^{3}$ and schools $s^{2}$ and $s^{3}$. But we do not have any information on how she for instance relates schools $s^{2}$ and $s^{4}$.

Now consider the mechanism DI. Obviously the induced game has a much richer structure than that of CI. In particular, strategies can be much more complex. And, in fact, by Claim 3, the preference strategy consistent with the true preferences need not be weakly dominant. For this reason, we expect at least as many switches - i.e., situations in which a student first proposes to a school $s^{j}$ and in a later step to a better school $s^{i}(i<j)$ - as in the case of CI, except that now we should take into account that there is only limited information about the student's strategy. More precisely, we will often not be able to determine whether a subject would make a switch at some point in the game, simply because we only observe one (possibly short) path of play.

HYPOTHESIS 1A: Subjects switch pairs weakly more often in treatment DI than in treatment CI.

\footnotetext{
${ }^{6}$ See, e.g., Calsamiglia et al. [5], Chen and Kesten [8], Chen and Sönmez [11], Ding and Schotter [12], Featherstone and Niederle [18], Guillen and Hakimov [23],[24], Guillen and Hing [25], Klijn et al. [32],[33], Pais and Pintér [35], and Zhu [44].
} 
Table 2 presents for each $i, j$ with $i<j$ in row $s^{i}-s^{j}$ the frequency with which we observe (the better) school $s^{i}$ is switched with (the worse) school $s^{j} .^{7}$

\begin{tabular}{|c|c|c|c|c|c|c|c|c|c|c|c|c|}
\hline & \multirow[t]{2}{*}{ CI } & \multicolumn{5}{|c|}{ Problem } & \multirow[t]{2}{*}{ DI } & \multicolumn{5}{|c|}{ Problem } \\
\hline & & All & 1 & 2 & 3 & 4 & & All & 1 & 2 & 3 & 4 \\
\hline$s^{1}-s^{2}$ & & 0.34 & 0.42 & 0.32 & 0.27 & 0.33 & & 0.33 & 0.35 & 0.33 & 0.32 & 0.35 \\
\hline$s^{1}-s^{3}$ & & 0.25 & 0.34 & 0.21 & 0.15 & 0.28 & & 0.14 & 0.17 & 0.08 & 0.07 & 0.25 \\
\hline$s^{1}-s^{4}$ & & 0.14 & 0.20 & 0.14 & 0.10 & 0.12 & & 0.07 & 0.08 & 0.01 & 0.01 & 0.17 \\
\hline$s^{2}-s^{3}$ & & 0.19 & 0.27 & 0.15 & 0.18 & 0.17 & & 0.16 & 0.19 & 0.09 & 0.09 & 0.28 \\
\hline$s^{2}-s^{4}$ & & 0.11 & 0.17 & 0.08 & 0.12 & 0.07 & & 0.09 & 0.12 & 0.01 & 0.02 & 0.21 \\
\hline$s^{3}-s^{4}$ & & 0.08 & 0.15 & 0.07 & 0.04 & 0.05 & & 0.24 & 0.23 & 0.05 & 0.17 & 0.29 \\
\hline Truth-telling & & 0.47 & 0.42 & 0.49 & 0.47 & 0.52 & & 0.55 & 0.52 & 0.63 & 0.63 & 0.43 \\
\hline
\end{tabular}

Table 2: Frequency that two schools are switched under the static (left) and dynamic (right) studentproposing DA mechanisms. Significant treatment effects obtained from panel data regressions with individual random effects (clustered on matching groups) are indicated in boldface for the treatment with the higher frequency. We also display the frequency of subjects who cannot be rejected to tell the truth.

The left part of the table shows that subjects often switch pairs in treatment CI. Also, in each of the four problems, the switching frequency is highest for the pair $s^{1}-s^{2}$ and lowest for the pair $s^{3}-s^{4}$. A comparison with the right part of the table reveals that switching frequencies have the tendency to be lower in treatment DI in Problems 1 and 2, leading to significant treatment effects for the pairs $s^{1}-s^{3}$ and $s^{1}-s^{4}$ in Problem 1 and for the pairs $s^{1}-s^{4}$ and $s^{2}-s^{4}$ in Problem 2. On the other hand, we can also see that the switching frequency is significantly higher in treatment DI than in treatment CI for the pair $s^{2}-s^{4}$ in Problem 4 and for the pair $s^{3}-s^{4}$ in Problems 3 and 4. Note that the significant differences in Problems 1 and 2 are mainly related to switches in which $s^{1}$ is reported below $s^{3}$ or $s^{4}$, while the significant differences in Problems 3 and 4 are related to switches in the lower part of the preferences (mainly interchanging $s^{3}$ and $s^{4}$ ). Finally, and with respect to the aggregate data, we find that the pair $s^{1}-s^{3}$ is switched significantly more often in treatment CI than in treatment DI, while the pair $s^{3}-s^{4}$ is switched significantly more often in treatment DI than in treatment CI.

Result 1A: In Problems 1 and 2, switching frequencies are significantly higher in treatment CI (than DI) for some pairs involving the top ranked schools. In Problems 3 and 4, switching frequencies are significantly higher in treatment DI (than CI) for some pairs involving the lowest ranked school.

\footnotetext{
${ }^{7}$ The empirical distribution of strategies in treatment CI can be found in Table 6 of Appendix B. Some strategies are pooled since there are $\left(\begin{array}{l}4 \\ 4\end{array}\right) \times 4 !+\left(\begin{array}{l}4 \\ 3\end{array}\right) \times 3 !+\left(\begin{array}{l}4 \\ 2\end{array}\right) \times 2 !+\left(\begin{array}{l}4 \\ 1\end{array}\right) \times 1 !+\left(\begin{array}{l}4 \\ 0\end{array}\right) \times 1 !=24+24+12+4+1=65$ strategies, many of which are not used (or only sporadically). It can be seen that truth-telling is the modal strategy and that deviations from truth-telling are not random in the sense that "Boston-style" reasoning (i.e., submitting the strategy $\left.s^{2}, s^{1}, s^{3}, s^{4}\right)$ is not uncommon.
} 
Table 2 also shows the frequencies with which subjects cannot be rejected to tell the truth. In treatment $\mathrm{CI}$ this is equal to determining the frequencies that subjects submit lists that coincide with their true preferences, while in treatment DI we calculate the frequencies of subjects whose observable behavior does not violate the play of a preference strategy consistent with truth-telling. Stated informally, we cannot reject truth-telling under DI whenever a subject "goes down the list" (a subject applies first to her most preferred school; then, if she gets rejected at some point of the game, an application to the second most preferred school is submitted; and so forth, until the game stops).

The results show that truth-telling rates are low in treatment CI. Moreover, comparing treatments CI and DI, we cannot reject the play of truth-telling more often in treatment DI than CI for Problems 1-3, which is due to two effects: subjects switch on average less pairs in these problems in treatment DI and frequencies are ex ante biased towards treatment DI since not all of the possible comparisons are observed in this treatment. ${ }^{8}$ In order to disentangle the two effects and make a fairer comparison between treatments CI and DI, we may consider just part of students' submitted lists in treatment CI. One possibility is to contemplate only a student's (true) list until the position of the highest ranked school (in the student's true preferences) who ranks the student first; in case such a school does not exist, consider a student's list until the position of the highest ranked school (in the student's true preferences) who ranks the student second; and, in case such schools do not exist, consider a student's list until the position of the highest ranked school (in the student's true preferences) who ranks the student third; otherwise, consider the full list. Following this approach, the percentages in treatment CI are $42 \%$ in Problem 1, 55\% in Problem 2, $53 \%$ in Problem 3, and 55\% in Problem $4 .^{9}$

\section{School-proposing DA mechanisms}

The static school-proposing mechanism differs from the static student-proposing mechanism in that students have now incentives to manipulate. In the dynamic implementation of the schoolproposing mechanism we would like to find out whether subjects understand that truth-telling might not always be optimal. More specifically, as a natural counterpart to Hypothesis 1A, we now seek to study whether and how a dynamic implementation of the school-proposing DA algorithm may change subjects' behavior.

Consider the mechanism CS. As mentioned in Section 3.4, a notable class of manipulations is that of strategies that involve a truncation of the true preferences. Obviously, truncation strategies have a very simple structure relative to the true preferences. But maybe more importantly, the

\footnotetext{
${ }^{8}$ Figure 3 of Appendix B regards the dynamics of truth-telling. More specifically, panels 1 and 3 depict the percentages with which subjects cannot be rejected to tell the truth for the student-proposing mechanisms. Some learning effects can be identified for the dynamic student-proposing mechanism.

${ }^{9}$ In all four problems this approach is equivalent to checking whether students truthfully list schools down to the school-optimal stable match. An alternative approach is to consider the percentages of telling the truth up to the student-optimal stable match, which leads to the percentages $51 \%$ in Problem 1, $62 \%$ in Problem $2,64 \%$ in Problem 3, and 55\% in Problem 4. As one could expect, the truth-telling rates in treatment CI get closer to those in DI by relaxing the notion of truth-telling. We are very grateful to an anonymous referee for these suggestions.
} 
set of truncation strategies is known to be strategically exhaustive, i.e., each school a student is matched to when manipulating her preferences can be obtained or strictly improved upon by using some truncation strategy (Roth and Vande Vate [39]). Truncation strategies have been observed in practice, for instance, in the sorority rush (Mongell and Roth [34]). Therefore, it is of interest to determine to which extent subjects employ truncation strategies in our experiment.

Now consider the mechanism DS. Similarly to the previous discussion on CI and DI, the mechanism DS induces a much richer game than CS. Additionally, according to Claim 6, the class of preference strategies consistent with truncations need not be strategically exhaustive. Therefore, when comparing the usage of truncation strategies in CS and in DS, we expect subjects to use preference strategies consistent with truncations weakly less often in DS than using actual truncations in CS. This is the content of Hypothesis 1B.

HYPOTHESIS 1B: Subjects act according to a truncation weakly less often in treatment DS than in treatment $C S$.

As previously noted, in the dynamic implementations of the mechanisms there is only limited information about students' strategies. In fact, we can only observe one path of play, so that in the case of DS we cannot tell whether a student's strategy is a preference strategy consistent with a truncation, i.e., if he would act according to a truncation of the true preferences for all possible paths of play. Therefore, in order to compare the usage of truncations under the school-proposing mechanisms, in treatment DS we consider the frequencies of subjects that cannot be rejected to play according to a truncation. This boils down to considering all cases in which the observable part of a student's strategy exhibits no switches. Note that this definition coincides with that of a truncation in CS, except for the fact that while in CS strategies are fully observable, in DS they are not. For example, in treatment DS, if a subject initially receives only an offer from $s^{2}$ that she accepts and rejects later on offers from $s^{3}$ and $s^{4}$, we can only conclude that the ordered list of preferences revealed by this subject in the play of the game includes $s^{2}$ and that $s^{2}$ is better ranked than both $s^{3}$ and $s^{4}$, but we do not know how $s^{3}$ and $s^{4}$ compare and whether these two schools would be accepted in case the subject was not holding $s^{2}$ 's proposal in the first place. Since there are many preference strategies consistent with a truncation that are not violated by the supposed behavior, we cannot reject that she behaves according to a truncation. This is different for a subject who rejects an offer from $s^{2}$ in the first step of the algorithm and temporarily accepts an offer from $s^{3}$ in the second step. Such a subject's strategy exhibits a switch and is clearly not a preference strategy consistent with a truncation of the true preferences.

In the last row of Table 3 we display the frequency of subjects who cannot be rejected to use a truncation for each problem. ${ }^{10}$ We see that only a few subjects can be rejected to play truncation strategies in treatment DS, while the frequencies of truncation under CS are much lower.

That there is more switching in treatment CS is also reflected in aggregate numbers. In fact, aggregated over all problems and pairs, we observe that the switching frequency in treatment CS

\footnotetext{
${ }^{10}$ Figure 3 of Appendix B regards the dynamics of truncating. More specifically, panels 2 and 4 depict the percentages with which subjects cannot be rejected to play according to a truncation strategy for the school-proposing mechanisms. Some learning effects can be identified for the static school-proposing mechanism in Problems 1-3.
} 


\begin{tabular}{|c|c|c|c|c|c|c|c|c|c|c|c|c|}
\hline & \multirow[t]{2}{*}{ CS } & \multicolumn{5}{|c|}{ Problem } & \multirow[t]{2}{*}{ DS } & \multicolumn{5}{|c|}{ Problem } \\
\hline & & All & 1 & 2 & 3 & 4 & & All & 1 & 2 & 3 & 4 \\
\hline$s^{1}$ & & 0.95 & 0.96 & 0.97 & 0.95 & 0.93 & & 0.99 & 0.99 & 0.98 & 1.00 & 1.00 \\
\hline$s^{2}$ & & 0.91 & 0.96 & 0.94 & 0.92 & 0.84 & & 0.84 & 0.84 & 0.74 & 0.94 & 1.00 \\
\hline$s^{3}$ & & 0.75 & 0.90 & 0.72 & 0.65 & 0.72 & & 0.55 & 0.57 & 0.51 & 0.46 & 0.92 \\
\hline$s^{4}$ & & 0.60 & 0.68 & 0.55 & 0.51 & 0.67 & & 0.27 & 0.37 & 0.02 & 0.02 & 0.77 \\
\hline Truncation & & 0.51 & 0.38 & 0.49 & 0.64 & 0.56 & & $0.9^{7}$ & 0.94 & 0.94 & 0.99 & 0.99 \\
\hline
\end{tabular}

Table 3: Frequency that a school is acceptable under the static (left) and dynamic (right) schoolproposing DA mechanisms. Significant treatment effects obtained from panel data regressions with individual random effects (clustered on matching groups) are indicated in boldface for the treatment with the higher frequency. We also display the frequency of subjects who cannot be rejected to use a truncation strategy.

is $19.6 \%$, much higher than in treatment DS, where merely $1.4 \%$ of all decisions involve switching pairs of schools. We thus find that under CS subjects switch pairs with frequencies that are similar to those presented in Table 2 for treatment CI, while under DS almost no subject accepts an offer from a school $s^{j}$ after having temporarily accepted an offer from a more preferred school $s^{i}(i<j) .{ }^{11}$ Additionally, in each problem, these differences between treatments CS and DS are significant for almost all pairs of schools. ${ }^{12}$

To further compare subjects' behavior in the two implementations of the school-proposing DA, in the upper part of Table 3 we compute the frequencies that an offer from a school is acceptable. More precisely, for CS we consider the frequency that a school is ranked in the submitted list. In the case of DS we proceed as follows. First, for each observed play of the game (induced by DS) and for each student $i$, we label each school $s$ "acceptable", "unacceptable", or "undetermined". School $s$ is labeled acceptable for $i$ if at some step of the procedure school $s$ makes an offer to $i$ and $i$ accepts it. School $s$ is labeled unacceptable for $i$ if at some step of the procedure school $s$ makes an offer to $i$, student $i$ does not retain some other school's offer, and $i$ rejects school $s$. Finally, school $s$ is labeled undetermined for $i$ otherwise. ${ }^{13}$ The frequencies for DS in Table 3 are the quotients (\#acceptable)/(\#acceptable + \#unacceptable), i.e., the cases $(i, s)$ where $s$ is undetermined for $i$ are excluded from the analysis.

The table shows that most schools are declared acceptable in the static school-proposing DA mechanism. Also, the data for this mechanism is consistent in the sense that for all $i<j$, the frequency that school $s^{i}$ is accepted is greater than or equal to the frequency with which school $s^{j}$ is accepted. This consistency can also be found in treatment DS, but, most importantly, the

\footnotetext{
${ }^{11}$ The empirical distribution of strategies in treatment CS can be found in Table 7 of Appendix B. Similarly to treatment CI, truth-telling is the modal strategy. Non-degenerate truncation strategies are not uncommon.

${ }^{12}$ We only do not observe a significant difference for pair $s^{1}-s^{2}$ in Problem 2.

${ }^{13}$ In the case of label "undetermined" either $i$ did not receive an offer from $s$ or $i$ did receive an offer from $s$ but accepted or kept an offer from some other school.
} 
acceptance rates of $s^{2}, s^{3}$, and $s^{4}$ are substantially lower in Problems 1-3, leading to significant treatment effects in many instances. Combined with low rates of switching under DS, this indicates that subjects' behavior is actually coherent with using a preference strategy consistent with a strict truncation, i.e., a truncation of the true preferences where a non-empty tail of less desired schools is removed.

Result 1B: Switching frequencies are significantly higher in treatment CS (than DS). Moreover, in Problems 1-3 subjects accept offers from low ranked schools significantly less often in treatment $D S$ (than $C S$ ).

\subsection{Stability}

Stability is the central solution concept in matching markets. In the problems we consider, a matching is said to be "blocked" if there is a student that prefers to be assigned to some school with a slot that is either available or occupied by another student with a lower priority. Then, a matching is "stable" if no student can form a blocking pair with any school. Problem 1 has four stable matchings. In the student-optimal stable matching, which is obtained in treatments CI and DI when all students reveal their preferences truthfully, each student is assigned to her top school. Similarly, the school-optimal stable matching, the matching obtained in treatments CS and DS under truth-telling, is such that each student is assigned to her worst school. In the two "compromise" stable matchings all students are assigned to their second most and third most preferred schools, respectively. Problem 2 has three and Problem 3 has two stable matchings. Finally, there is one stable matching in Problem 4 (where for each $k=1, \ldots, 4$, student $i_{k}$ is matched to school $s_{k}$ ) and all four mechanisms coincide under truth-telling.

There is no reason to expect that stability is higher under the dynamic implementations of the mechanisms. In fact, according to Claim 2, all Nash equilibrium outcomes in CI (which includes all stable and possibly some unstable matchings) can also be sustained in Nash equilibrium in the game induced by DI. Moreover, according to Claim 4, the set of Nash equilibrium outcomes of the game induced by DS is the set of stable matchings, which hence coincides with the set of Nash equilibrium outcomes in CS.

HYPOTHESIS 2: The frequency of stable matchings is weakly lower under the dynamic implementations than in their static counterparts.

Nevertheless, we have already seen that the likelihood of switching pairs of schools in the studentproposing mechanisms and of behaving in a way consistent with a (strict) truncation of the true preferences in the school-proposing mechanisms may depend on whether we consider the static or the dynamic implementations. We now analyze whether and how these different behaviors affect the rates of stable outcomes.

Figure 1 plots the frequency with which a stable matching is reached over the course of the experiment. ${ }^{14}$

\footnotetext{
${ }^{14}$ Figure 4 of Appendix B focuses on the frequency of the student-optimal stable matching. One important
} 

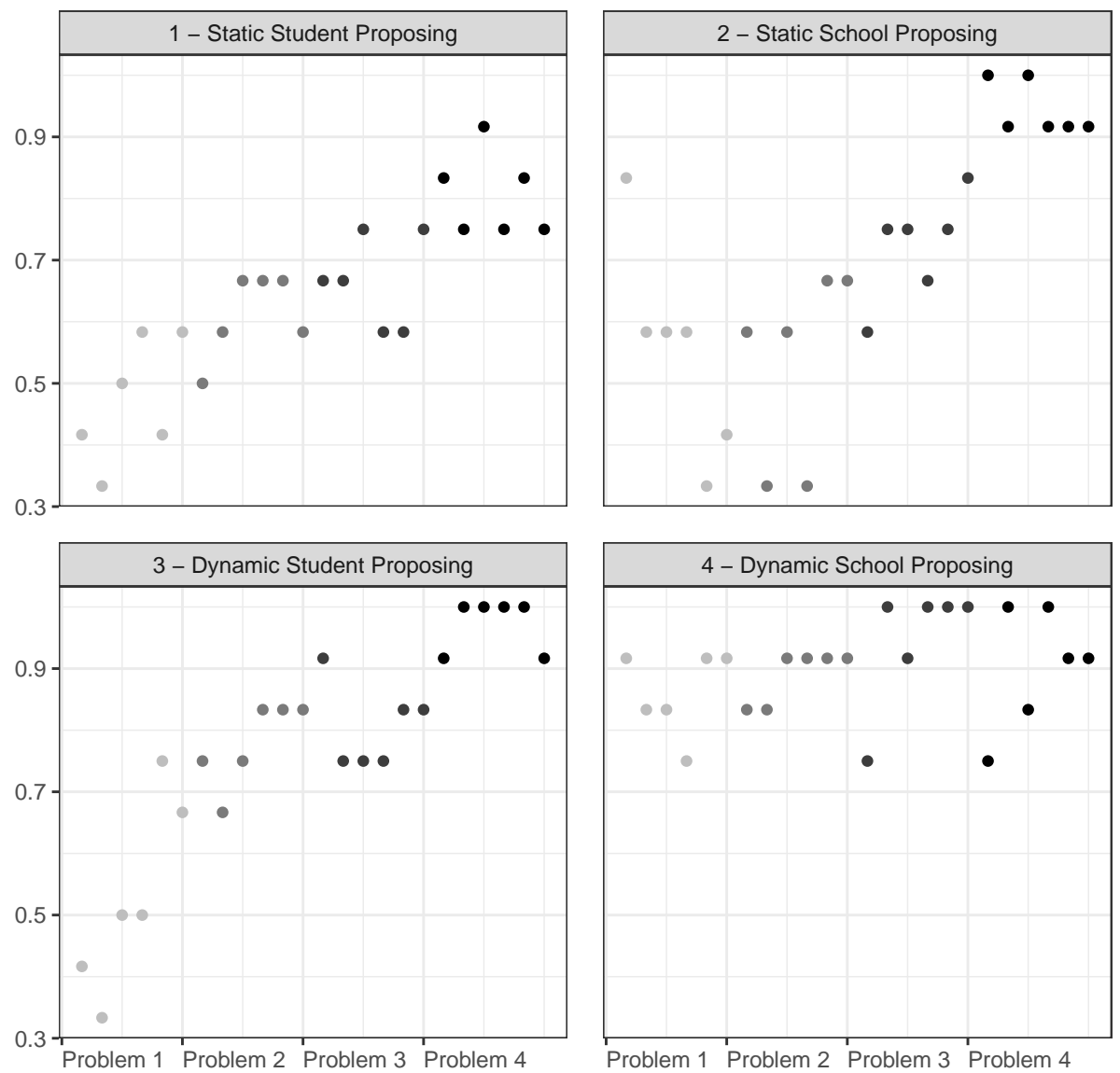

Figure 1: Frequency of stable matchings.

There are stark differences between the treatments. ${ }^{15}$ The dynamic school-proposing DA mechanism seems to perform best among all four mechanisms. In Problems 1-3 it visibly outclasses not only its natural peer, the static school-proposing DA mechanism, but also the two studentproposing DA mechanisms. On the other hand, possible differences between the two studentproposing DA mechanisms are less pronounced. This intuition is confirmed if we look at the overall frequencies of stable matchings (63.88\% in treatment CI, $77.08 \%$ in treatment DI, $68.75 \%$ in treatment CS, and $89.93 \%$ in treatment DS).

We run four random effects estimations, one for each problem, to assess treatment differences (Table 4). The dependent variable $y_{i}$ is equal to 1 if the resulting matching in group $i$ is stable, and 0 if it is unstable. Our explanatory variables are three dummy variables that identify the

take away from this analysis is that in Problems 1 and 2, the student-optimal matching is only rarely obtained in treatments CI, DI, and CS, but quite often in treatment DS. Moreover, the (qualitative) main differences between the mechanisms that can be observed in Figure 4 are similar to those in Figure 1. We are very grateful to an anonymous referee for suggesting this different metric.

${ }^{15}$ Note that since for each $k \in\{1,2,3,4\}$, Problem $k$ has $5-k$ stable matchings, comparisons across problems should be made with caution. 
different treatments: the variable "Student-proposing" $\left(\beta_{2}\right)$ takes value 1 in treatments CI and DI and 0 in treatments CS and DS, the variable "Static" $\left(\beta_{3}\right)$ takes value 1 in treatments CI and CS and 0 in treatments DI and DS, and the variable "Student-proposing $\times$ Static" $\left(\beta_{4}\right)$ takes value 1 in treatment CI and 0 in the other three treatments. Since each problem is played six times, we also include the variable "Repetition" (which takes values from 1 to 6) in order to account for learning effects within a problem. In this setting, differences across treatments are evaluated against treatment DS, which serves as a benchmark. In particular, $\beta_{3}$ measures the differences between treatments CS and DS, while $\beta_{3}+\beta_{4}$ assesses the differences between CI and DI.

\begin{tabular}{lcccc}
\hline \hline & Problem 1 & Problem 2 & Problem 3 & Problem 4 \\
\hline Constant $\left(\beta_{0}\right)$ & $0.8361^{* * *}$ & $0.8014^{* * *}$ & $0.8861^{* * *}$ & $0.9049^{* * *}$ \\
& $(0.0976)$ & $(0.1054)$ & $(0.1018)$ & $(0.0612)$ \\
Repetition $\left(\beta_{1}\right)$ & 0.0071 & $0.0250^{*}$ & 0.0167 & -0.0006 \\
& $(0.0141)$ & $(0.0107)$ & $(0.0087)$ & $(0.0084)$ \\
Student-proposing $\left(\beta_{2}\right)$ & $-0.3333^{* *}$ & -0.1111 & -0.1389 & 0.0694 \\
& $(0.1192)$ & $(0.1392)$ & $(0.1374)$ & $(0.0758)$ \\
Static $\left(\beta_{3}\right)$ & $-0.3056^{*}$ & $-0.3611^{* *}$ & -0.2222 & 0.0417 \\
& $(0.1192)$ & $(0.1392)$ & $(0.1374)$ & $(0.0758)$ \\
Student-proposing $\times$ Static $\left(\beta_{4}\right)$ & 0.2500 & 0.1944 & 0.0833 & -0.2083 \\
& $(0.1686)$ & $(0.1969)$ & $(0.1943)$ & $(0.1072)$ \\
\hline $\mathrm{R}^{2}$ & 0.0444 & 0.0458 & 0.0286 & 0.0193 \\
Observations & 288 & 288 & 288 & 288 \\
\hline \hline
\end{tabular}

Table 4: Random effects estimations on the frequency of reaching a stable matching. Errors are clustered on matching groups. ${ }^{* * *}$ indicates significance at $p=0.001$ (two-sided), ${ }^{* *}$ indicates significance at $p=0.01$ (two-sided), and ${ }^{*}$ indicates significance at $p=0.05$ (two-sided). The qualitative insights remain the same if we employ probit estimations.

In consistence with Figure 1, the value of $\beta_{3}$ in Table 4 is significantly smaller than 0 in Problems 1 and 2; that is, the likelihood of reaching a stable matching in treatment DS is higher than in treatment CS (by 31 percentage points in Problem 1 and 36 percentage points in Problem 2). Also, $\beta_{2}$ is significantly smaller than 0 in Problem 1. Hence, in this problem, the frequency of reaching a stable matching is higher in treatment DS than in treatment DI. Next, we apply $\chi^{2-}$ tests to see whether the values of $\beta_{3}+\beta_{4}$ or $\beta_{2}+\beta_{4}$ are significantly different from zero. The only significant effect is found in Problem 4, where the unique stable matching is reached more frequently in treatment DI than in treatment CI at $p=0.05$. Putting all results together, we observe that in all problems (some problem) the frequency of reaching a stable matching under a dynamic mechanism is at least as high as (strictly higher than) under its static counterpart. This allows us to conclude as follows. ${ }^{16}$

\footnotetext{
${ }^{16}$ An alternative way of assessing stability consists in calculating the percentage of (all possible) student-school pairs that can block a resulting matching. The corresponding percentages in (Problem 1, Problem 2, Problem 3,
} 
Result 2: Each dynamic mechanism weakly outperforms its static counterpart in terms of stability.

\subsection{Welfare}

For the dynamic mechanisms to be reasonable alternatives for the standard static mechanisms, it is crucial that decisions under the former mechanisms also translate into higher payoffs. And, given the results above that compare the set of Nash equilibrium outcomes in the dynamic and static mechanisms, there is a priori no reason to expect that the dynamic implementations present an improvement over their static counterparts in this dimension. In fact, switching the order of schools is a weakly dominated strategy in CI and the unique Nash equilibrium outcome in undominated strategies in this mechanism is the student-optimal stable matching, which maximizes total payoffs in all problems. By contrast, there are no weakly dominant strategies in DI and the set of equilibrium outcomes is a superset of the set of stable matchings (Claim 2), thus containing inefficient matchings. On the other hand, in what the school-proposing mechanisms are concerned, by Claim 4, the set of Nash equilibrium outcomes in both mechanisms equals the set of stable matchings. Moreover, if all subjects use truncation strategies in CS — which are known to be strategically exhaustive under this mechanism - a stable matching is obtained as long as subjects do not truncate above their schools under the student-optimal stable matching. In fact, by truncating, stable matchings that correspond to higher average payoffs may be obtained.

HYPOTHESIS 3: Average payoffs are weakly lower under the dynamic implementations than under their static counterparts.

Nevertheless, with respect to the student-proposing mechanisms, we have seen in Result $1 \mathrm{~A}$ that only the pairs $s^{2}-s^{4}$ and $s^{3}-s^{4}$ are switched sometimes more often in treatment DI than in treatment CI (in Problems 3 and 4). The fact that subjects actually switch less (or act according to the true preferences more often) under DI, may imply that the student-optimal stable matching is obtained more often than what theoretic results imply, so that welfare levels may be higher than expected. And, with respect to the school-proposing DA mechanisms, there are only very few subjects who can be rejected to play a preference strategy consistent with a truncation of the true preferences in treatment DS compared to treatment CS (Result 1B). Again, this may imply that subjects obtain schools that are ranked higher in their preference lists and, thus, welfare levels in the dynamic mechanism are higher than what theoretical results would suggest.

Figure 2 plots the average payoffs over the course of the experiment. Since it is not always possible to assign all students to their most preferred school, comparisons across problems should be made with caution. We can make two main observations. First, in Problems 1 and 2, each dynamic implementation leads to higher payoffs than its static counterpart, the difference being

Problem 4) are $(4.43 \%, 3.21 \%, 2.25 \%, 1.56 \%)$ in treatment CI, $(3.65 \%, 1.48 \%, 1.21 \%, 0.26 \%)$ in treatment DI, $(4.77 \%, 5.82 \%, 3.73 \%, 1.56 \%)$ in treatment CS, and $(2.86 \%, 3.73 \%, 2.17 \%, 2.17 \%)$ in treatment DS. The only instance where a static mechanism has a lower percentage than the corresponding dynamic mechanism occurs in Problem 4 for the school-proposing mechanism. 

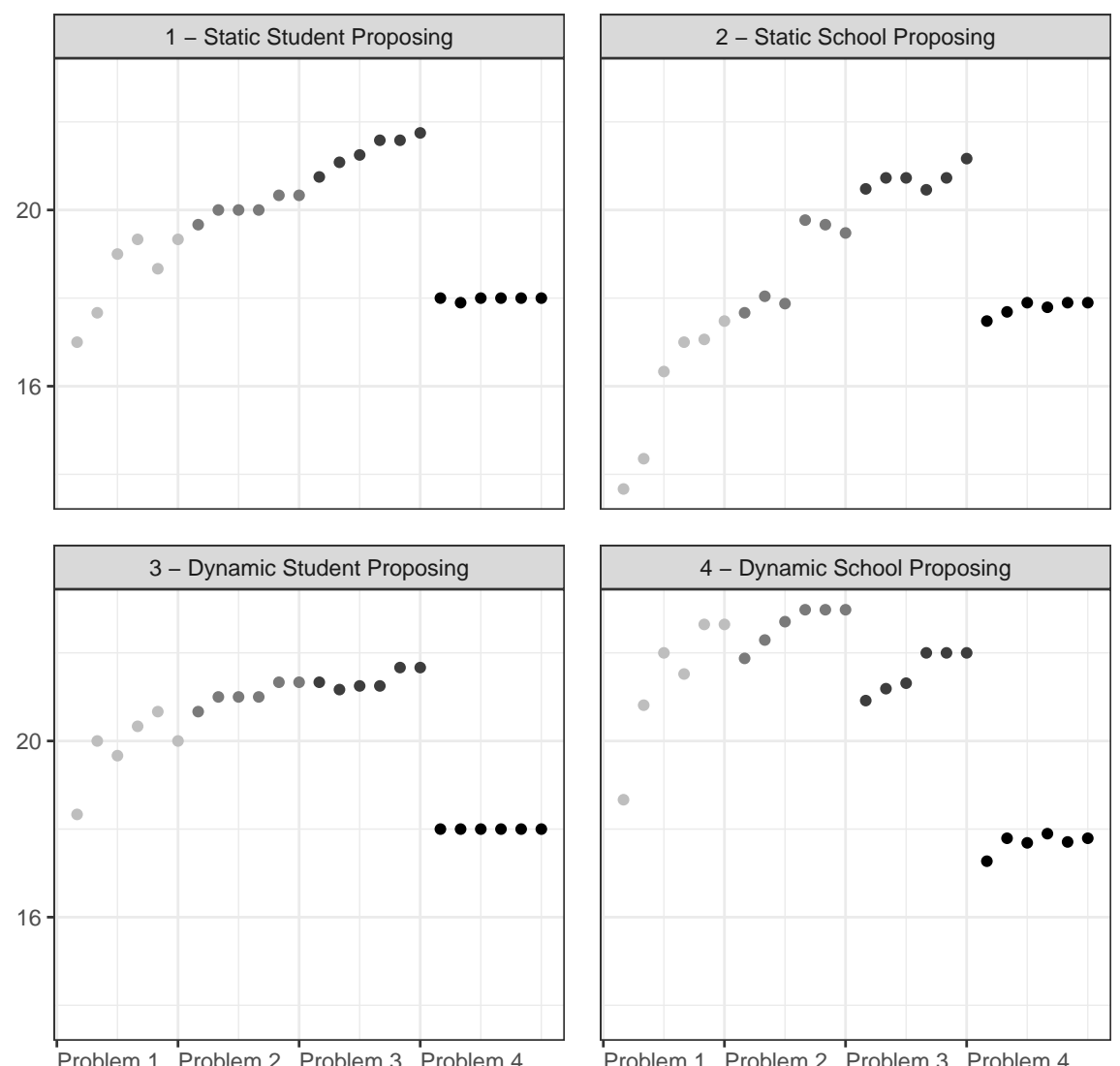

Figure 2: Average payoff per round in each of the four treatments. The student-(school-)optimal stable matching leads to an average payoff of 24 ECU (12 ECU) in Problem 1, 24 ECU (16 ECU) in Problem 2, 22 ECU (16 ECU) in Problem 3, and 18 ECU (18 ECU) in Problem 4.

more pronounced for the school-proposing mechanism. Second, treatment differences are small in Problem 3 and negligible in Problem 4. Finally, aggregated over all problems, average payoffs are 19.47 ECU in treatment CI, 20.07 ECU in treatment DI, 18.31 ECU in treatment CS, and 20.82 ECU in treatment DS. This is suggestive evidence that the dynamic implementation of the school-proposing DA mechanism performs not only better than the static school-proposing DA mechanism, but also does not yield lower payoffs than the two student-proposing mechanisms. To see whether these visual insights are confirmed, we run again random effects regressions (Table 5), using the same explanatory variables as in the subsection on stability and taking the payoff of subject $i$ as the dependent variable $y_{i}$.

Supporting the conclusions drawn from the observation of Figure 2, the estimated payoffs of a subject in Problems 3 and 4 are the same in all four treatments (21.03 ECU in Problem 3 and 17.55 ECU in Problem 4). They are close to the expected payoffs associated with the student-optimal stable matching, namely 22 ECU in Problem 3 and 18 ECU in Problem 4. More importantly, we can see that in Problems 1 and 2, both $\beta_{2}$ and $\beta_{3}$ are negative and significantly different from 


\begin{tabular}{|c|c|c|c|c|}
\hline & Problem 1 & Problem 2 & Problem 3 & Problem 4 \\
\hline Constant $\left(\beta_{0}\right)$ & $\begin{array}{l}19.4127^{* * *} \\
(0.3628)\end{array}$ & $\begin{array}{l}21.8266^{* * *} \\
(0.3679)\end{array}$ & $\begin{array}{l}21.0267^{* * *} \\
(0.3949)\end{array}$ & $\begin{array}{l}17.5540^{* * *} \\
(0.6671)\end{array}$ \\
\hline Repetition $\left(\beta_{1}\right)$ & $\begin{array}{l}0.5626^{* * *} \\
(0.0494)\end{array}$ & $\begin{array}{l}0.2311^{* * *} \\
(0.0364)\end{array}$ & $\begin{array}{l}0.1551^{* * *} \\
(0.0312)\end{array}$ & $\begin{array}{c}0.0391 \\
(0.0243)\end{array}$ \\
\hline Student-proposing $\left(\beta_{2}\right)$ & $\begin{array}{c}-1.5486^{* * *} \\
(0.4511)\end{array}$ & $\begin{array}{c}-1.5799^{* * *} \\
(0.4881)\end{array}$ & $\begin{array}{c}-0.1806 \\
(0.5366)\end{array}$ & $\begin{array}{c}0.3090 \\
(0.9357)\end{array}$ \\
\hline Static $\left(\beta_{3}\right)$ & $\begin{array}{c}-5.3993^{* * *} \\
(0.4511)\end{array}$ & $\begin{array}{c}-3.8854^{* * *} \\
(0.4881)\end{array}$ & $\begin{array}{c}-0.8542 \\
(0.5366)\end{array}$ & $\begin{array}{c}0.0833 \\
(0.9357)\end{array}$ \\
\hline Student-proposing $\times$ Static $\left(\beta_{4}\right)$ & $\begin{array}{l}4.0660^{* * * *} \\
(0.6379)\end{array}$ & $\begin{array}{l}2.8854^{* * *} \\
(0.6903)\end{array}$ & $\begin{array}{c}0.7986 \\
(0.7589)\end{array}$ & $\begin{array}{c}-0.1007 \\
(1.3233)\end{array}$ \\
\hline $\mathrm{R}^{2}$ & 0.1985 & 0.0861 & 0.0235 & 0.0024 \\
\hline Observations & 1152 & 1152 & 1152 & 1152 \\
\hline
\end{tabular}

Table 5: Random effects estimations on the average payoff. Errors are clustered on matching groups. *** indicates significance at $p=0.001$ (two-sided), ${ }^{* *}$ indicates significance at $p=0.01$ (two-sided), and * indicates significance at $p=0.05$ (two-sided).

zero at $p=0.001$; that is, payoffs in these problems are higher in treatment DS than in both treatment DI and treatment CS. Finally, we apply $\chi^{2}$-tests to see whether there are differences between treatments CI and DI and between treatments CI and CS. We find for both problems at $p=0.05$ that payoffs are significantly higher in treatment DI than in treatment CI, which, in turn, yields significantly higher payoffs than treatment CS.

Result 3: Each dynamic mechanism weakly outperforms the two static mechanisms in terms of payoffs.

\section{Conclusion}

Abdulkadiroğlu and Sönmez [1] show that the student-proposing DA mechanism has nice theoretical properties in that revealing one's preferences truthfully is a weakly dominant strategy in the induced static game and the student-optimal stable matching is obtained as a consequence. However, when the mechanism is brought to the laboratory it is commonly found that untrained subjects behave strategically even though they should not. It is thus the objective of this paper to analyze whether better experimental outcomes can be obtained if, instead of submitting lists, subjects make decisions dynamically by going through each of the underlying matching algorithms.

Our theoretical results for the dynamic student-proposing DA mechanism show that if players use a "going down the list" strategy, that is, they take a ranking of schools (that may or may not coincide with their true preferences) and always send an application to the better ranked school 
they have not yet applied to, then the outcome is equal to the outcome of the static game when the corresponding lists are submitted to the central planner. But since the dynamic game allows for richer strategies, it is not true that going down the list with respect to one's true preferences is a weakly dominant strategy, so that in a laboratory experiment a wider range of behavior could be expected under the dynamic implementation. We nevertheless find experimentally that subjects tend to switch fewer pairs of schools at the top of their true preferences under the dynamic implementation of the student-proposing DA mechanism, positively affecting outcomes in terms of stability (63.88\% in the static and $77.08 \%$ in the dynamic mechanism) and average payoffs (19.47 ECU in the static and 20.07 ECU in the dynamic mechanism).

We also consider the static and the dynamic school-proposing DA mechanisms. Students typically have incentives to manipulate the static mechanism, for instance by using truncation strategies, which are strategically exhaustive in this context. We have theoretically shown that in the search for best replies under the dynamic mechanism it may not be sufficient to restrict attention to preference strategies based on truncations of the the true preferences. So, a wider range of behavior can again be supported for the dynamic implementation. Perhaps surprisingly, we find in our laboratory experiment that the dynamic school-proposing DA mechanism does not only outperform the corresponding static implementation in terms of percentage of stable matchings (68.75\% in the static and $89.93 \%$ in the dynamic mechanism) and efficiency (18.31 ECU in the static and 20.82 ECU in the dynamic mechanism), but also the two student-proposing DA mechanisms. The reason for this lies in changes of subjects' behavior. First, if an offer from some school is temporarily accepted under the dynamic implementation, subjects do not switch that school for a worse one later on, which is crucial for preventing unstable outcomes to occur. The overall switching frequency in the static implementation, on the other hand, is with $20 \%$ rather high and close to that of the static student-proposing DA mechanism. Second, it is only under the dynamic school-proposing DA mechanism that subjects learn that it can be worthwhile in terms of payoffs to reject offers from less desirable schools.

Our experimental results have some policy implications. In most real-life applications, the static student-proposing DA mechanism is employed. However, our findings show that in this case the potential improvement from a dynamic implementation could be slightly limited and hence might not constitute a fully satisfactory alternative. Even more so if we take into account possible complications (time and organization) in order to actually carry out a dynamic implementation.

On the positive side, there are also real-life applications where the static school-proposing DA mechanism is used (see Footnote 1). For these markets, our results show that a transition to a dynamic implementation could lead to substantial gains in terms of efficiency and stability. We believe that it is not unlikely that such a change would be supported by their participants. Not only because of potential welfare gains that we highlighted, but also because a step-bystep implementation could actually make the underlying algorithm more transparent. In any case, one would still have to deal with potential practical problems that would bring along the implementation of such a dynamic mechanism.

Surprisingly, the dynamic school-proposing DA mechanism is the best performing mechanism in our study (at least in terms of stability and average payoffs). We do not suggest this mechanism 
be implemented in real-life applications where the current mechanism is not the static schoolproposing DA mechanism. The reason is twofold. First, participants may have other concerns (for instance, whether a school-proposing DA mechanism would actually not disfavor them, or would (even more) unlevel the playing field). Second, such a more radical departure from current practice is likely to be prone to objections from policy-makers and participants alike. 


\section{References}

[1] A Abdulkadiroğlu and T Sönmez (2003). School choice: A mechanism design approach. American Economic Review 93(3): 729-747.

[2] I Bó and R Hakimov (2016). Iterative versus standard deferred acceptance: Experimental evidence. Working Paper, WZB Berlin Social Science Center. https://ssrn.com/abstract= 2860738

[3] I Bó and R Hakimov (2017). The iterative deferred acceptance mechanism. Working Paper, WZB Berlin Social Science Center. https://ssrn.com/abstract=2881880

[4] S Braun, N Dwenger, and D Kübler (2010). Telling the truth may not pay off: An empirical study of centralized university admissions in Germany. The B.E. Journal of Economic Analysis E) Policy Vol. 10: Iss. 1 (Advances), Article 22.

[5] C Calsamiglia, G Haeringer, and F Klijn (2010). Constrained school choice: An experimental study. American Economic Review 100(4): 1860-1874.

[6] M Castillo and A Dianat (2016). Truncation strategies in two-sided matching markets: Theory and experiment. Games and Economic Behavior 98: 180-196.

[7] M Castillo and A Dianat (2016). Information effects in the dynamic Gale-Shapley mechanism: An experimental study. Working Paper, Interdisciplinary Center for Economic Science, George Mason University.

[8] Y Chen and O Kesten (2016). Chinese college admissions and school choice reforms: An experimental study. Working Paper, School of Information, University of Michigan.

[9] Y Chen and O Kesten (2017). Chinese college admissions and school choice reforms: A theoretical analysis. Journal of Political Economy 125(1): 99-139.

[10] L Chen and J Pereyra (2017). Time-constrained school choice. Working Paper, ECARES.

[11] Y Chen and T Sönmez (2006). School choice: An experimental study. Journal of Economic Theory 127(1): 202-231.

[12] T Ding and A Schotter (2017). Matching and chatting: An experimental study of the impact of network communication on school-matching mechanisms. Games and Economic Behavior 103: $94-115$.

[13] L Dubins and D Freedman (1981). Machiavelli and the Gale-Shapley algorithm. American Mathematical Monthly 88(7): 485-494.

[14] U Dur, R Hammond, and T Morrill (2017). Sequential school choice: Theory and evidence from the field and lab. Working Paper, North Carolina State University. 
[15] U Dur, R Hammond, and T Morrill (2018). Identifying the harm of manipulable school-choice mechanisms. American Economic Journal: Economic Policy, 10(1): 187-213.

[16] F Echenique, A Wilson, and L Yariv (2016). Clearinghouses for two-sided matching: An experimental study. Quantitative Economics 7(2): 449-482.

[17] C Featherstone and E Mayefsky (2015). Why do some clearinghouses yield stable outcomes? Experimental evidence on out-of-equilibrium truth-telling. Working Paper, The Wharton School, University of Pennsylvania.

[18] C Featherstone and M Niederle (2016). Boston versus deferred acceptance in an interim setting: An experimental investigation. Games and Economic Behavior 100: 353-375.

[19] U Fischbacher (2007). z-Tree: Zurich toolbox for ready-made economic experiments. Experimental Economics 10(2): 171-178.

[20] D Gale and L Shapley (1962). College admissions and the stability of marriage. American Mathematical Monthly 69(1): 9-15.

[21] D Gale and M Sotomayor (1985). Ms Machiavelli and the stable matching problem. American Mathematical Monthly 92(4): 261-268.

[22] B Gong and Y Liang (2016). A dynamic college admission mechanism in Inner Mongolia: Theory and experiment. Working Paper, School of Information, University of Michigan.

[23] P Guillen and R Hakimov (2015). How to get truthful reporting in matching markets: A field experiment. WZB Discussion Paper, No. SP II 2015-208.

[24] P Guillen and R Hakimov (2017). Not quite the best response: truth-telling, strategy-proof matching, and the manipulation of others. Experimental Economics 20(3): 670-686.

[25] P Guillen and A Hing (2014). Lying through their teeth: Third party advice and truth telling in a strategy proof mechanism. European Economic Review 70(1): 178-185.

[26] G Haeringer and F Klijn (2009). Constrained school choice. Journal of Economic Theory 144(5): 1921-1947.

[27] E Haruvy and U Ünver (2007). Equilibrium selection and the role of information in repeated matching markets. Economics Letters 94(2): 284-289.

[28] A Hassidim, A Romm, and R Shorrer (2018). "Strategic" behavior in a strategy-proof environment. Working Paper, https://ssrn.com/abstract=2784659

[29] V Hiller and O Tercieux (2013). Matching practices for secondary schools-France. Matching in Practice Network, http://www.matching-in-practice.eu/matching-practices-in-secondaryschools-france/ 
[30] V Hiller and O Tercieux (2014). Choix d'écoles en France. Une évaluation de la procédure Affelnet. Revue Économique 65(3): 619-656.

[31] P Jaramillo, Ç Kayı, and F Klijn (2014). On the exhaustiveness of truncation and dropping strategies in many-to-many matching markets. Social Choice and Welfare 42(4): 793-811.

[32] F Klijn, J Pais, and M Vorsatz (2013). Preference intensities and risk aversion in school choice: A laboratory experiment. Experimental Economics 16(1): 1-22.

[33] F Klijn, J Pais, and M Vorsatz (2016). Affirmative action through minority reserves: An experimental study on school choice. Economics Letters 139: 72-75.

[34] S Mongell and A Roth (1991). Sorority rush as a two-sided matching mechanism. American Economic Review 81(3): 441-464.

[35] J Pais and Á Pintér (2008). School choice and information: An experimental study on matching mechanisms. Games and Economic Behavior 64(1): 303-328.

[36] A Rees-Jones (2017). Suboptimal behavior in strategy-proof mechanisms: Evidence from the residency match. Games and Economic Behavior, forthcoming.

[37] A Roth (1982). The economics of matching: Stability and incentives. Mathematics of Operations Research 7(4): 617-628.

[38] A Roth (1984). Misrepresentation and stability in the marriage problem. Journal of Economic Theory 34(2): 383-387.

[39] A Roth and J Vande Vate (1991). Incentives in two-sided matching with random stable mechanisms. Economic Theory 1(1): 31-44.

[40] A Roth and X Xing (1997). Turnaround time and bottlenecks in market clearing: Decentralized matching in the market for clinical psychologists. Journal of Political Economy 105(2): 284-329.

[41] M Salonen (2014). Matching practices for secondary schools-Finland. Matching in Practice Network, http://www.matching-in-practice.eu/matching-practices-for-secondary-schoolsfinland/

[42] C Terrier (2014). Matching practices of teachers to schools - France. Matching in Practice Network, http://www.matching-in-practice.eu/matching-practices-of-teachers-to-schools-france/

[43] A Westkamp (2013). An analysis of the German university admissions system. Economic Theory 53(3): 561-589.

[44] M Zhu (2015). Experience transmission: Truth-telling adoption in matching. Working Paper, Université Lumière (Lyon 2). https://ssrn.com/abstract=2631442 


\section{Appendix A, proofs (intended for online publication)}

\section{Proof of Claim 2}

Let $Q$ be a Nash equilibrium in the game induced by CI leading to $\mu$. Let $\sigma$ be the profile of preference strategies consistent with $Q$ in the game induced by DI. By Claim 1 and Remark 1, $\sigma$ leads to $\mu$. We show that $\sigma$ is a Nash equilibrium of the game induced by DI. By contradiction, assume that $\hat{\sigma}_{i}$ is a profitable deviation for some student $i$ in the game induced by DI and let $\hat{Q}_{i}$ be the ordered list that is revealed by student $i$ in the play of $\left(\hat{\sigma}_{i}, \sigma_{-i}\right)$. By Claim 1, the outcome of the game induced by DI when students use $\left(\hat{\sigma}_{i}, \sigma_{-i}\right)$ coincides with the outcome of the game induced by CI when students submit $\left(\hat{Q}_{i}, Q_{-i}\right)$. Therefore, $\hat{Q}_{i}$ is a profitable deviation for $i$ against $Q_{-i}$ in the game induced by CI, which contradicts the initial assumption that $Q$ is a Nash equilibrium in the game induced by CI.

\section{Proof of Claim 3}

\section{Problem 1}

We show that the preference strategy for $i_{1}$ that is consistent with her true preferences is not a weakly dominant strategy. Let $\sigma_{-i_{1}}$ be a preference profile of students $\left\{i_{2}, i_{3}, i_{4}\right\}$ such that

(1) if $j=i_{2}$ then $\sigma_{j}$ prescribes to apply to $s_{2}$ in the first phase and whenever she gets rejected by $s_{2}$ to apply to $s_{3}$ (when rejection by $s_{2}$ takes place in first phase) and $s_{1}$ (when rejection by $s_{2}$ takes place in second or later phase);

(2) if $j=i_{3}$ then $\sigma_{j}$ is the preference strategy consistent with $Q_{i_{3}}=s_{1}, s_{2}$;

(3) if $j=i_{4}$ then $\sigma_{j}$ is the preference strategy consistent with $Q_{i_{4}}=s_{4}$.

Let $\sigma_{i_{1}}$ be the preference strategy consistent with $Q_{i_{1}}=s_{2}$. Obviously, $\sigma_{i_{1}}$ is not consistent with the true preferences $P_{i_{1}}$. Next, we compute the matching obtained in the game induced by DI when the students play strategy profile $\sigma$. Notation: $i \rightarrow s$ indicates that student $i$ makes an offer to school $s$.

Phase 1:

offers: $i_{1}, i_{2} \rightarrow s_{2}, i_{3} \rightarrow s_{1}$ and $i_{4} \rightarrow s_{4}$

tentative matching: $i_{1}-s_{2}, i_{3}-s_{1}, i_{4}-s_{4}$

Phase 2:

offers: $i_{2} \rightarrow s_{3}$

final matching: $i_{1}-s_{2}, i_{2}-s_{3}, i_{3}-s_{1}, i_{4}-s_{4}$

Let $\sigma_{i_{1}}^{\prime}$ be consistent with the true preferences $P_{i_{1}}=s_{1}, s_{2}, s_{3}, s_{4}$. We compute the matching obtained in the game induced by DI when the students play strategy profile $\sigma^{\prime} \equiv\left(\sigma_{i_{1}}^{\prime}, \sigma_{-i_{1}}\right)$.

Phase 1:

offers: $i_{1}, i_{3} \rightarrow s_{1}, i_{2} \rightarrow s_{2}$ and $i_{4} \rightarrow s_{4}$

tentative matching: $i_{2}-s_{2}, i_{3}-s_{1}, i_{4}-s_{4}$ 
Phase 2:

offers: $i_{1} \rightarrow s_{2}$

tentative matching: $i_{1}-s_{2}, i_{3}-s_{1}, i_{4}-s_{4}$

Phase 3:

offers: $i_{2} \rightarrow s_{1}$

tentative matching: $i_{1}-s_{2}, i_{2}-s_{1}, i_{4}-s_{4}$

Phase 4:

offers: $i_{3} \rightarrow s_{2}$

tentative matching: $i_{2}-s_{1}, i_{3}-s_{2}, i_{4}-s_{4}$

Phase 5:

offers: $i_{1} \rightarrow s_{3}$

final matching: $i_{1}-s_{3}, i_{2}-s_{1}, i_{3}-s_{2}, i_{4}-s_{4}$

Since $i_{1}$ prefers $s_{2}$ (obtained at $\sigma$ ) to $s_{3}$ (obtained at $\sigma^{\prime}$ ), the preference strategy for $i_{1}$ consistent with her true preferences is not a weakly dominant strategy in the extensive-form game.

\section{Problem 2}

We show that the preference strategy for $i_{3}$ that is consistent with her true preferences is not a weakly dominant strategy. Let $\sigma_{-i_{3}}$ be a preference profile of students $\left\{i_{1}, i_{2}, i_{4}\right\}$ such that

(1) if $j=i_{1}$ then $\sigma_{j}$ is the preference strategy consistent with $Q_{i_{1}}=s_{4}, s_{1}$;

(2) if $j=i_{2}$ then $\sigma_{j}$ is the preference strategy consistent with $Q_{i_{2}}=s_{2}$;

(3) if $j=i_{4}$ then $\sigma_{j}$ prescribes to apply to $s_{1}$ in the first phase and whenever she gets rejected by $s_{1}$ to apply to $s_{3}$ (when rejection by $s_{1}$ takes place in first phase) and $s_{4}$ (when rejection by $s_{1}$ takes place in second or later phase).

Let $\sigma_{i_{3}}$ be the preference strategy consistent with $Q_{i_{3}}=s_{1}$. Obviously, $\sigma_{i_{3}}$ is not consistent with the true preferences $P_{i_{3}}$. Next, we compute the matching obtained in the game induced by DI when the students play strategy profile $\sigma$. Notation: $i \rightarrow s$ indicates that student $i$ makes an offer to school $s$.

Phase 1:

offers: $i_{1} \rightarrow s_{4}, i_{2} \rightarrow s_{2}$ and $i_{3}, i_{4} \rightarrow s_{1}$

tentative matching: $i_{1}-s_{4}, i_{2}-s_{2}, i_{3}-s_{1}$

Phase 2:

offers: $i_{4} \rightarrow s_{3}$

final matching: $i_{1}-s_{4}, i_{2}-s_{2}, i_{3}-s_{1}, i_{4}-s_{3}$

Let $\sigma_{i_{3}}^{\prime}$ be consistent with the true preferences $P_{i_{3}}=s_{4}, s_{1}, s_{2}, s_{3}$. We compute the matching obtained in the game induced by DI when the students play strategy profile $\sigma^{\prime} \equiv\left(\sigma_{i_{3}}^{\prime}, \sigma_{-i_{3}}\right)$.

Phase 1:

offers: $i_{1}, i_{3} \rightarrow s_{4}, i_{2} \rightarrow s_{2}$ and $i_{4} \rightarrow s_{1}$

tentative matching: $i_{1}-s_{4}, i_{2}-s_{2}, i_{4}-s_{1}$ 
Phase 2:

offers: $i_{3} \rightarrow s_{1}$

tentative matching: $i_{1}-s_{4}, i_{2}-s_{2}, i_{3}-s_{1}$

Phase 3:

offers: $i_{4} \rightarrow s_{4}$

tentative matching: $i_{2}-s_{2}, i_{3}-s_{1}, i_{4}-s_{4}$

Phase 4:

offers: $i_{1} \rightarrow s_{1}$

tentative matching: $i_{1}-s_{1}, i_{2}-s_{2}, i_{4}-s_{4}$

Phase 5:

offers: $i_{3} \rightarrow s_{2}$

tentative matching: $i_{1}-s_{1}, i_{2}-s_{2}, i_{4}-s_{4}$

Phase 6:

offers: $i_{3} \rightarrow s_{3}$

final matching: $i_{1}-s_{1}, i_{2}-s_{2}, i_{3}-s_{3}, i_{4}-s_{4}$

Since $i_{3}$ prefers $s_{1}$ (obtained at $\sigma$ ) to $s_{3}$ (obtained at $\sigma^{\prime}$ ), the preference strategy for $i_{3}$ consistent with her true preferences is not a weakly dominant strategy in the extensive-form game.

\section{Problem 3}

We show that the preference strategy for $i_{1}$ that is consistent with her true preferences is not a weakly dominant strategy. Let $\sigma_{-i_{1}}$ be a preference profile of students $\left\{i_{2}, i_{3}, i_{4}\right\}$ such that

(1) if $j=i_{2}$ then $\sigma_{j}$ prescribes to apply to $s_{2}$ in the first phase and whenever she gets rejected by $s_{2}$ to apply to $s_{4}$ (when rejection by $s_{2}$ takes place in first phase) and $s_{3}$ (when rejection by $s_{2}$ takes place in second or later phase);

(2) if $j=i_{3}$ then $\sigma_{j}$ is the preference strategy consistent with $Q_{i_{3}}=s_{4}, s_{1}$;

(3) if $j=i_{4}$ then $\sigma_{j}$ is the preference strategy consistent with $Q_{i_{4}}=s_{4}, s_{2}$.

Let $\sigma_{i_{1}}$ be the preference strategy consistent with $Q_{i_{1}}=s_{1}$. Obviously, $\sigma_{i_{1}}$ is not consistent with the true preferences $P_{i_{1}}$. Next, we compute the matching obtained in the game induced by DI when the students play strategy profile $\sigma$. Notation: $i \rightarrow s$ indicates that student $i$ makes an offer to school $s$.

Phase 1:

offers: $i_{1} \rightarrow s_{1}, i_{2} \rightarrow s_{2}$ and $i_{3}, i_{4} \rightarrow s_{4}$

tentative matching: $i_{1}-s_{1}, i_{2}-s_{2}, i_{3}-s_{4}$

Phase 2:

offers: $i_{4} \rightarrow s_{2}$

tentative matching: $i_{1}-s_{1}, i_{3}-s_{4}, i_{4}-s_{2}$ 
Phase 3:

offers: $i_{2} \rightarrow s_{3}$

final matching: $i_{1}-s_{1}, i_{2}-s_{3}, i_{3}-s_{4}, i_{4}-s_{2}$

Let $\sigma_{i_{1}}^{\prime}$ be consistent with the true preferences $P_{i_{1}}=s_{2}, s_{1}, s_{3}, s_{4}$. We compute the matching obtained in the game induced by DI when the students play strategy profile $\sigma^{\prime} \equiv\left(\sigma_{i_{1}}^{\prime}, \sigma_{-i_{1}}\right)$.

Phase 1:

offers: $i_{1}, i_{2} \rightarrow s_{2}$ and $i_{3}, i_{4} \rightarrow s_{4}$

tentative matching: $i_{1}-s_{2}, i_{3}-s_{4}$

Phase 2:

offers: $i_{2} \rightarrow s_{4}$ and $i_{4} \rightarrow s_{2}$

tentative matching: $i_{2}-s_{4}, i_{4}-s_{2}$

Phase 3:

offers: $i_{1}, i_{3} \rightarrow s_{1}$

tentative matching: $i_{2}-s_{4}, i_{3}-s_{1}, i_{4}-s_{2}$

Phase 4:

offers: $i_{1} \rightarrow s_{3}$

final matching: $i_{1}-s_{3}, i_{2}-s_{4}, i_{3}-s_{1}, i_{4}-s_{2}$

Since $i_{1}$ prefers $s_{1}$ (obtained at $\sigma$ ) to $s_{3}$ (obtained at $\sigma^{\prime}$ ), the preference strategy for $i_{1}$ consistent with her true preferences is not a weakly dominant strategy in the extensive-form game.

\section{Problem 4}

We show that the preference strategy for $i_{3}$ that is consistent with her true preferences is not a weakly dominant strategy. Let $\sigma_{-i_{3}}$ be a preference profile of students $\left\{i_{1}, i_{2}, i_{4}\right\}$ such that

(1) if $j=i_{1}$ then $\sigma_{j}$ is the preference strategy consistent with $Q_{i_{1}}=s_{4}$;

(2) if $j=i_{2}$ then $\sigma_{j}$ prescribes to apply to $s_{1}$ in the first phase and whenever she gets rejected by $s_{1}$ to apply to $s_{2}$ (when rejection by $s_{1}$ takes place in first phase) and $s_{3}$ (when rejection by $s_{1}$ takes place in second or later phase);

(3) if $j=i_{4}$ then $\sigma_{j}$ is the preference strategy consistent with $Q_{i_{4}}=s_{4}, s_{1}$.

Let $\sigma_{i_{3}}$ be the preference strategy consistent with $Q_{i_{3}}=s_{2}$. Obviously, $\sigma_{i_{3}}$ is not consistent with the true preferences $P_{i_{3}}$. Next, we compute the matching obtained in the game induced by DI when the students play strategy profile $\sigma$. Notation: $i \rightarrow s$ indicates that student $i$ makes an offer to school $s$.

Phase 1:

offers: $i_{1}, i_{4} \rightarrow s_{4}, i_{2} \rightarrow s_{1}$ and $i_{3} \rightarrow s_{2}$

tentative matching: $i_{1}-s_{4}, i_{2}-s_{1}, i_{3}-s_{2}$

Phase 2:

offers: $i_{4} \rightarrow s_{1}$

tentative matching: $i_{1}-s_{4}, i_{3}-s_{2}, i_{4}-s_{1}$ 
Phase 3:

offers: $i_{2} \rightarrow s_{3}$

final matching: $i_{1}-s_{4}, i_{2}-s_{3}, i_{3}-s_{2}, i_{4}-s_{1}$

Let $\sigma_{i_{3}}^{\prime}$ be consistent with the true preferences $P_{i_{3}}=s_{1}, s_{2}, s_{3}, s_{4}$. We compute the matching obtained in the game induced by DI when the students play strategy profile $\sigma^{\prime} \equiv\left(\sigma_{i_{3}}^{\prime}, \sigma_{-i_{3}}\right)$.

Phase 1:

offers: $i_{1}, i_{4} \rightarrow s_{4}$ and $i_{2}, i_{3} \rightarrow s_{1}$

tentative matching: $i_{1}-s_{4}, i_{3}-s_{2}$

Phase 2:

offers: $i_{2} \rightarrow s_{2}$ and $i_{4} \rightarrow s_{1}$

tentative matching: $i_{1}-s_{4}, i_{2}-s_{2}, i_{4}-s_{1}$

Phase 3:

offers: $i_{3} \rightarrow s_{2}$

tentative matching: $i_{1}-s_{4}, i_{2}-s_{2}, i_{4}-s_{1}$

Phase 4:

offers: $i_{3} \rightarrow s_{3}$

final matching: $i_{1}-s_{4}, i_{2}-s_{2}, i_{3}-s_{3}, i_{4}-s_{1}$

Since $i_{3}$ prefers $s_{2}$ (obtained at $\sigma$ ) to $s_{3}$ (obtained at $\sigma^{\prime}$ ), the preference strategy for $i_{3}$ consistent with her true preferences is not a weakly dominant strategy in the extensive-form game.

\section{Proof of Claim 4}

Let us start by showing that every equilibrium outcome is stable. By contradiction, let a matching $\mu \notin S(P)$ be reached in equilibrium $\sigma$. Since all schools are acceptable for all students in the problems we consider, this means that there exists a blocking pair, i.e., a pair $(s, i)$ such that $s P_{i} \mu(i)$ and $i P_{s} \mu(s)$. Given that proposals are issued according to the schools' preferences, it must have been the case that $i$ received school $s$ 's proposal and rejected it at some point. Therefore, consider a strategy $\sigma_{i}^{\prime}$ for student $i$ that coincides with the strategy $\sigma_{i}$, except for the fact that once $s^{\prime}$ 's proposal is received, it is held and every other proposal is rejected. Using this strategy against the strategies of the other students $\sigma_{-i}$ guarantees that $i$ is matched to $s$ (the game proceeds in exactly the same way as before up to the point when student $i$ does not reject the offer from $s$ and thus ends up matched to $s)$. Since $s P_{i} \mu(i)$, the strategy $\sigma_{i}^{\prime}$ is a profitable deviation for student $i$ and $\sigma$ is not an equilibrium.

To show that every matching $\mu \in S(P)$ can be reached in equilibrium consider the strategy profile $\sigma$ where each student $i$ holds $\mu(i)$ 's proposal whenever received and rejects every other proposal. Clearly, $\sigma$ leads to $\mu$. Moreover, there is no profitable deviation for any student $i$. In fact, for every $s \in S$ such that $s P_{i} \mu(i)$, by stability of $\mu$, we have $\mu(s) P_{s} i$. Hence, by definition of $\sigma_{-i}, s$ proposes $\mu(s)$ before proposing $i$ and $\mu(s)$ holds this proposal and ends up matched to $s$. 


\section{Proof of Claim 6}

\section{Problem 1}

Let $i=i_{4}$. Let $\sigma_{-i}$ be a preference profile of students $\left\{i_{1}, i_{2}, i_{3}\right\}$ such that for any information set $\mathcal{I} \in \Im_{j}$ with $j \neq i, \sigma_{j}$ prescribes that

(1) if $j=i_{1}$ and $j$ receives only an offer from $s_{4}$, she keeps $s_{4}$;

if $j=i_{1}$ retains an offer from $s_{4}$ and receives an offer from $s_{2}$ without having received an offer from $s_{3}$ previously, she keeps $s_{2}$ (and rejects $s_{4}$ );

if $j=i_{1}$ retains an offer from $s_{4}$ and receives an offer from $s_{2}$ after having received an offer from $s_{3}$, she rejects $s_{2}$ (and keeps $s_{4}$ );

if $j=i_{1}$ retains an offer from $s_{4}$ and receives an offer from $s_{3}$, she rejects the offer from $s_{3}$ (and keeps $\left.s_{4}\right)$;

(2) if $j=i_{2}$ and $j$ receives only an offer from $s_{1}, s_{2}$, or $s_{4}$, she rejects the offer;

if $j=i_{2}$ receives an offer from $s_{3}$, she accepts it;

(3) if $j=i_{3}$ and $j$ receives only an offer from $s_{1}$, she accepts $s_{1}$;

if $j=i_{3}$ receives an offer from $s_{2}$ or $s_{4}$, she rejects the offer.

Let $\sigma_{i}$ be a strategy such that in any information set $\mathcal{I} \in \Im_{i}, \sigma_{i}$ prescribes that

(4a) if $i$ receives only an offer from $s_{3}$, she keeps the offer from $s_{3}$;

(4b) if $i$ retains an offer from $s_{3}$ and receives only an offer from $s_{2}$, she rejects it (and keeps the offer from $\left.s_{3}\right)$;

(4c) if $i$ retains an offer from $s_{3}$ and receives only an offer from $s_{4}$, she accepts it (and rejects the offer from $s_{3}$ ).

Since $s_{2} P_{i} s_{3}$, it follows from $(4 \mathrm{~b})$ that $\sigma_{i}$ is not a preference strategy consistent with a truncation of the true preferences. Next, we compute the matching obtained in the game induced by DS when the students play strategy profile $\sigma$. Notation: $s \rightarrow i$ indicates that school $s$ makes an offer to student $i$.

Phase 1:

offers: $s_{1} \rightarrow i_{2}, s_{2}, \rightarrow i_{3}, s_{3}, \rightarrow i_{4}$, and $s_{4} \rightarrow i_{1}$

tentative matching: $i_{1}-s_{4}, i_{4}-s_{3}$

Phase 2:

offers: $s_{1} \rightarrow i_{3}$ and $s_{2} \rightarrow i_{4}$

tentative matching: $i_{1}-s_{4}, i_{3}-s_{1}, i_{4}-s_{3}$

Phase 3:

offers: $s_{2} \rightarrow i_{1}$

tentative matching: $i_{1}-s_{2}, i_{3}-s_{1}, i_{4}-s_{3}$ 
Phase 4:

offers: $s_{4} \rightarrow i_{2}$

tentative matching: $i_{1}-s_{2}, i_{3}-s_{1}, i_{4}-s_{3}$

Phase 5:

offers: $s_{4} \rightarrow i_{3}$

tentative matching: $i_{1}-s_{2}, i_{3}-s_{1}, i_{4}-s_{3}$

Phase 6:

offers: $s_{4} \rightarrow i_{4}$

tentative matching: $i_{1}-s_{2}, i_{3}-s_{1}, i_{4}-s_{4}$

Phase 7:

offers: $s_{3} \rightarrow i_{1}$

tentative matching: $i_{1}-s_{2}, i_{3}-s_{1}, i_{4}-s_{4}$

Phase 8:

offers: $s_{3} \rightarrow i_{2}$

final matching: $i_{1}-s_{2}, i_{2}-s_{3}, i_{3}-s_{1}, i_{4}-s_{4}$

Note that at $\sigma$ student $i_{4}$ is matched to her most preferred school.

Let $\sigma_{i}^{\prime}$ be any preference strategy consistent with a truncation $P_{i}^{t}$ of student $i$ 's true preferences $P_{i}=s_{4}, s_{1}, s_{2}, s_{3}$. We compute the matching obtained in the game induced by DS when the students play strategy profile $\sigma^{\prime} \equiv\left(\sigma_{i}^{\prime}, \sigma_{-i}\right)$. We distinguish between three cases.

CASE 1: $i$ uses $P_{i}=s_{4}, s_{1}, s_{2}, s_{3}$.

Phase 1:

offers: $s_{1} \rightarrow i_{2}, s_{2}, \rightarrow i_{3}, s_{3}, \rightarrow i_{4}$, and $s_{4} \rightarrow i_{1}$

tentative matching: $i_{1}-s_{4}, i_{4}-s_{3}$

Phase 2:

offers: $s_{1} \rightarrow i_{3}$ and $s_{2} \rightarrow i_{4}$

tentative matching: $i_{1}-s_{4}, i_{3}-s_{1}, i_{4}-s_{2}$

Phase 3:

offers: $s_{3} \rightarrow i_{1}$

tentative matching: $i_{1}-s_{4}, i_{3}-s_{1}, i_{4}-s_{2}$

Phase 4:

offers: $s_{3} \rightarrow i_{2}$

final matching: $i_{1}-s_{4}, i_{2}-s_{3}, i_{3}-s_{1}, i_{4}-s_{2}$

CASE 2: $s_{3}$ is not acceptable in the truncation used by $i$, i.e., $P_{i}^{t}=s_{4}, s_{1}, s_{2}$.

Phase 1:

offers: $s_{1} \rightarrow i_{2}, s_{2}, \rightarrow i_{3}, s_{3}, \rightarrow i_{4}$, and $s_{4} \rightarrow i_{1}$

tentative matching: $i_{1}-s_{4}$

Phase 2:

offers: $s_{1} \rightarrow i_{3}, s_{2} \rightarrow i_{4}$, and $s_{3} \rightarrow i_{1}$

tentative matching: $i_{1}-s_{4}, i_{3}-s_{1}, i_{4}-s_{2}$ 
Phase 3:

offers: $s_{3} \rightarrow i_{2}$

final matching: $i_{1}-s_{4}, i_{2}-s_{3}, i_{3}-s_{1}, i_{4}-s_{2}$

CASE 3: $s_{2}$ and $s_{3}$ are not acceptable in the truncation used by i, i.e., $P_{i}^{t}=\emptyset, P_{i}^{t}=s_{4}$, or $P_{i}^{t}=s_{4}, s_{1}$.

Phase 1:

offers: $s_{1} \rightarrow i_{2}, s_{2}, \rightarrow i_{3}, s_{3}, \rightarrow i_{4}$, and $s_{4} \rightarrow i_{1}$

tentative matching: $i_{1}-s_{4}$

Phase 2:

offers: $s_{1} \rightarrow i_{3}, s_{2} \rightarrow i_{4}$, and $s_{3} \rightarrow i_{1}$

tentative matching: $i_{1}-s_{4}, i_{3}-s_{1}$

Phase 3:

offers: $s_{2} \rightarrow i_{1}$ and $s_{3} \rightarrow i_{2}$

tentative matching: $i_{1}-s_{4}, i_{2}-s_{3}, i_{3}-s_{1}$

Phase 4:

offers: $s_{2} \rightarrow i_{2}$

final matching: $i_{1}-s_{4}, i_{2}-s_{3}, i_{3}-s_{1}$

In each of the three cases, student $i_{4}$ is not matched to her most preferred school, proving the claim for Problem 1.

\section{Problem 2}

Let $i=i_{4}$. Let $\sigma_{-i}$ be a preference profile of students $\left\{i_{1}, i_{2}, i_{3}\right\}$ such that for any information set $\mathcal{I} \in \Im_{j}$ with $j \neq i, \sigma_{j}$ prescribes that

(1) if $j=i_{1}$ and $j$ receives only an offer from $s_{1}$, she rejects $s_{1}$;

if $j=i_{1}$ and $j$ receives only an offer from $s_{4}$, she accepts $s_{4}$;

if $j=i_{1}$ receives only an offer from both $s_{1}$ and $s_{4}$, she keeps the offer from $s_{1}$ (and rejects the offer from $s_{4}$ );

(2) if $j=i_{2}$ and $j$ receives only an offer from both $s_{1}$ and $s_{2}$, she keeps the offer from $s_{2}$ (and rejects the offer from $s_{1}$ );

if $j=i_{2}$ receives an offer from $s_{4}$, she rejects it;

(3) if $j=i_{3}$ and $j$ receives only an offer from $s_{3}$, she accepts $s_{3}$;

if $j=i_{3}$ receives an offer from $s_{1}$, she rejects it;

if $j=i_{3}$ receives an offer from $s_{4}$, she rejects it.

Let $\sigma_{i}$ be a strategy such that in any information set $\mathcal{I} \in \Im_{i}, \sigma_{i}$ prescribes that

(4a) if $i$ receives only an offer from both $s_{3}$ and $s_{4}$, she keeps the offer from $s_{4}$ (and rejects the offer from $\left.s_{3}\right)$; 
(4b) if $i$ retains a school and receives only an offer from $s_{1}$, she keeps it (and rejects any other offer).

Since $s_{3} P_{i} s_{4}$, it follows from (4a) that $\sigma_{i}$ is not a preference strategy consistent with a truncation of the true preferences. Next, we compute the matching obtained in the game induced by DS when the students play strategy profile $\sigma$. Notation: $s \rightarrow i$ indicates that school $s$ makes an offer to student $i$.

Phase 1:

offers: $s_{1}, s_{2} \rightarrow i_{2}$ and $s_{3}, s_{4} \rightarrow i_{4}$

tentative matching: $i_{2}-s_{2}, i_{4}-s_{4}$

Phase 2:

offers: $s_{1} \rightarrow i_{1}$ and $s_{3} \rightarrow i_{3}$

tentative matching: $i_{2}-s_{2}, i_{3}-s_{3}, i_{4}-s_{4}$

Phase 3:

offers: $s_{1} \rightarrow i_{3}$

tentative matching: $i_{2}-s_{2}, i_{3}-s_{3}, i_{4}-s_{4}$

Phase 4:

offers: $s_{1} \rightarrow i_{4}$

tentative matching: $i_{2}-s_{2}, i_{3}-s_{3}, i_{4}-s_{1}$

Phase 5:

offers: $s_{4} \rightarrow i_{1}$

final matching: $i_{1}-s_{4}, i_{2}-s_{2}, i_{3}-s_{3}, i_{4}-s_{1}$

Note that at $\sigma$ student $i_{4}$ is matched to her most preferred school.

Let $\sigma_{i}^{\prime}$ be any preference strategy consistent with a truncation $P_{i}^{t}$ of student $i$ 's true preferences $P_{i}=s_{1}, s_{2}, s_{3}, s_{4}$. We compute the matching obtained in the game induced by DS when the students play strategy profile $\sigma^{\prime} \equiv\left(\sigma_{i}^{\prime}, \sigma_{-i}\right)$. We distinguish between two cases.

CASE 1: $s_{3}$ is acceptable in the truncation used by $i$.

Phase 1:

offers: $s_{1}, s_{2} \rightarrow i_{2}$ and $s_{3}, s_{4} \rightarrow i_{4}$

tentative matching: $i_{2}-s_{2}, i_{4}-s_{3}$

Phase 2:

offers: $s_{1}, s_{4} \rightarrow i_{1}$

tentative matching: $i_{1}-s_{1}, i_{2}-s_{2}, i_{4}-s_{3}$

Phase 3:

offers: $s_{4} \rightarrow i_{2}$

tentative matching: $i_{1}-s_{1}, i_{2}-s_{2}, i_{4}-s_{3}$

Phase 4:

offers: $s_{4} \rightarrow i_{3}$

final matching: $i_{1}-s_{1}, i_{2}-s_{2}, i_{4}-s_{3}$ 
CASE 2: $s_{3}$ is not acceptable in the truncation used by $i$, i.e., $P_{i}^{t}=\emptyset, P_{i}^{t}=s_{1}$, or $P_{i}^{t}=s_{1}, s_{2}$. Phase 1:

offers: $s_{1}, s_{2} \rightarrow i_{2}$ and $s_{3}, s_{4} \rightarrow i_{4}$

tentative matching: $i_{2}-s_{2}$

Phase 2:

offers: $s_{1}, s_{4} \rightarrow i_{1}$ and $s_{3} \rightarrow i_{3}$

tentative matching: $i_{1}-s_{1}, i_{2}-s_{2}, i_{3}-s_{3}$

Phase 3:

offers: $s_{4} \rightarrow i_{2}$

tentative matching: $i_{1}-s_{1}, i_{2}-s_{2}, i_{3}-s_{3}$

Phase 4:

offers: $s_{4} \rightarrow i_{3}$

final matching: $i_{1}-s_{1}, i_{2}-s_{2}, i_{3}-s_{3}$

In each of the two cases, student $i_{4}$ is not matched to her most preferred school, proving the claim for Problem 2.

\section{Problem 3}

Let $i=i_{4}$. Let $\sigma_{-i}$ be a preference profile of students $\left\{i_{1}, i_{2}, i_{3}\right\}$ such that for any information set $\mathcal{I} \in \Im_{j}$ with $j \neq i, \sigma_{j}$ prescribes that

(1) if $j=i_{1}$ and $j$ receives only an offer from $s_{3}$, she keeps it;

if $j=i_{1}$ and $j$ receives only an offer from $s_{4}$, she rejects it;

if $j=i_{1}$ and $j$ receives only an offer from both $s_{3}$ and $s_{4}$, she keeps the offer from $s_{4}$ (and rejects the offer from $s_{3}$ );

(2) if $j=i_{2}$ receives or retains an offer from $s_{1}$, she keeps it (and rejects any other offer);

(3) if $j=i_{3}$ receives or retains an offer from $s_{2}$, she keeps it (and rejects any other offer).

Let $\sigma_{i}$ be a strategy such that in any information set $\mathcal{I} \in \Im_{i}, \sigma_{i}$ prescribes that

(4a) if $i$ does not retain a school and receives only an offer from both $s_{2}$ and $s_{3}$, she keeps the offer from $s_{3}$ (and rejects the offer from $s_{2}$ );

(4b) if $i$ retains or receives only an offer from $s_{4}$, she keeps it (and rejects any other offer).

Since $s_{2} P_{i} s_{3}$, it follows from (4a) that $\sigma_{i}$ is not a preference strategy consistent with a truncation of the true preferences. Next, we compute the matching obtained in the game induced by DS when the students play strategy profile $\sigma$.

Phase 1:

offers: $s_{1}, s_{4} \rightarrow i_{2}$ and $s_{2}, s_{3} \rightarrow i_{4}$

tentative matching: $i_{2}-s_{1}, i_{4}-s_{3}$ 
Phase 2:

offers: $s_{2} \rightarrow i_{3}$ and $s_{4} \rightarrow i_{1}$

tentative matching: $i_{2}-s_{1}, i_{3}-s_{2}, i_{4}-s_{3}$

Phase 3:

offers: $s_{4} \rightarrow i_{3}$

tentative matching: $i_{2}-s_{1}, i_{3}-s_{2}, i_{4}-s_{3}$

Phase 4:

offers: $s_{4} \rightarrow i_{4}$

tentative matching: $i_{2}-s_{1}, i_{3}-s_{2}, i_{4}-s_{4}$

Phase 5:

offers: $s_{3} \rightarrow i_{1}$

final matching: $i_{1}-s_{3}, i_{2}-s_{1}, i_{3}-s_{2}, i_{4}-s_{4}$

Note that at $\sigma$ student $i_{4}$ is matched to her most preferred school.

Let $\sigma_{i}^{\prime}$ be a preference strategy consistent with a truncation $P_{i}^{t}$ of student $i$ 's true preferences $P_{i}=s_{4}, s_{1}, s_{2}, s_{3}$. We compute the matching obtained in the game induced by DS when the students play strategy profile $\sigma^{\prime} \equiv\left(\sigma_{i}^{\prime}, \sigma_{-i}\right)$. We distinguish between two cases.

CASE 1: $P_{i}^{t}=s_{4}, s_{1}, s_{2}$ or $P_{i}^{t}=s_{4}, s_{1}, s_{2}, s_{3}$.

Phase 1:

offers: $s_{1}, s_{4} \rightarrow i_{2}$ and $s_{2}, s_{3} \rightarrow i_{4}$

tentative matching: $i_{2}-s_{1}, i_{4}-s_{2}$

Phase 2:

offers: $s_{3} \rightarrow i_{1}$ and $s_{4} \rightarrow i_{1}$

tentative matching: $i_{1}-s_{4}, i_{2}-s_{1}, i_{4}-s_{2}$

Phase 3:

offers: $s_{3} \rightarrow i_{2}$

tentative matching: $i_{1}-s_{4}, i_{2}-s_{1}, i_{4}-s_{2}$

Phase 4:

offers: $s_{3} \rightarrow i_{3}$

final matching: $i_{1}-s_{4}, i_{2}-s_{1}, i_{4}-s_{2}$ or $i_{1}-s_{4}, i_{2}-s_{1}, i_{3}-s_{3}, i_{4}-s_{2}$

CASE 2: $P_{i}^{t}=\emptyset, P_{i}^{t}=s_{4}$, or $P_{i}^{t}=s_{4}, s_{1}$.

Phase 1:

offers: $s_{1}, s_{4} \rightarrow i_{2}$ and $s_{2}, s_{3} \rightarrow i_{4}$

tentative matching: $i_{2}-s_{1}$

Phase 2:

offers: $s_{2} \rightarrow i_{3}$ and $s_{3}, s_{4} \rightarrow i_{1}$

tentative matching: $i_{1}-s_{4}, i_{2}-s_{1}, i_{3}-s_{2}$

In the remainder of the algorithm $s_{3}$ will only make an offer to students $i_{2}$ and $i_{3}$. However, according to their strategies, students $i_{2}$ and $i_{3}$ will retain their schools $s_{1}$ and $s_{2}$. Hence, the final matching is given by $i_{1}-s_{4}, i_{2}-s_{1}, i_{3}-s_{2}$. 
In each of the two cases, student $i_{4}$ is not matched to her most preferred school, which completes the proof of the claim for Problem 3.

\section{Problem 4}

Let $i=i_{2}$. Let $\sigma_{-i}$ be a preference profile of students $\left\{i_{1}, i_{3}, i_{4}\right\}$ such that for any information set $\mathcal{I} \in \Im_{j}$ with $j \neq i, \sigma_{j}$ prescribes that

(1) if $j=i_{1}$ and $j$ receives offers from all schools, she keeps $s_{4}$ (and rejects all other offers);

(2) if $j=i_{3}$ and $j$ receives only an offer from $s_{1}$, she rejects it;

if $j=i_{3}$ receives an offer from $s_{3}$, she keeps it;

if $j=i_{3}$ receives only an offer from both $s_{1}$ and $s_{3}$, she keeps the offer from $s_{1}$ (and rejects the offer from $\left.s_{3}\right)$;

(3) if $j=i_{4}$ and $j$ receives only an offer from $s_{1}$, she rejects it;

if $j=i_{4}$ receives an offer from $s_{2}$, she keeps it;

if $j=i_{4}$ receives only an offer from $s_{3}$, she keeps it;

if $j=i_{4}$ receives an offer from $s_{3}$ when retaining $s_{2}$, she rejects it (and keeps $s_{2}$ ).

Let $\sigma_{i}$ be a strategy such that in any information set $\mathcal{I} \in \Im_{i}, \sigma_{i}$ prescribes that

(4a) if $i$ does not retain a school and receives only an offer from both $s_{2}$ and $s_{3}$, she keeps the offer from $s_{3}$ (and rejects the offer from $s_{2}$ );

(4b) if $i$ retains a school and receives only an offer from $s_{1}$, she keeps it (and rejects any other offer).

Since $s_{2} P_{i} s_{3}$, it follows from (4a) that $\sigma_{i}$ is not a preference strategy consistent with a truncation of the true preferences. Next, we compute the matching obtained in the game induced by DS when the students play strategy profile $\sigma$.

Phase 1:

offers: $s_{1}, \ldots, s_{4} \rightarrow i_{1}$

tentative matching: $i_{1}-s_{4}$

Phase 2:

offers: $s_{1} \rightarrow i_{4}$ and $s_{2}, s_{3} \rightarrow i_{2}$

tentative matching: $i_{1}-s_{4}, i_{2}-s_{3}$

Phase 3:

offers: $s_{1} \rightarrow i_{3}$ and $s_{2} \rightarrow i_{4}$

tentative matching: $i_{1}-s_{4}, i_{2}-s_{3}, i_{4}-s_{2}$

Phase 4:

offers: $s_{1} \rightarrow i_{2}$

tentative matching: $i_{1}-s_{4}, i_{2}-s_{1}, i_{4}-s_{2}$ 
Phase 5:

offers: $s_{3} \rightarrow i_{3}$

final matching: $i_{1}-s_{4}, i_{2}-s_{1}, i_{3}-s_{3}, i_{4}-s_{2}$

Note that at $\sigma$ student $i_{2}$ is matched to her most preferred school.

Let $\sigma_{i}^{\prime}$ be any preference strategy consistent with a truncation $P_{i}^{t}$ of student $i$ 's true preferences $P_{i}=s_{1}, s_{2}, s_{3}, s_{4}$. We compute the matching obtained in the game induced by DS when the students play strategy profile $\sigma^{\prime} \equiv\left(\sigma_{i}^{\prime}, \sigma_{-i}\right)$. We distinguish between two cases.

CASE 1: $s_{2}$ is acceptable in the truncation used by $i$.

Phase 1:

offers: $s_{1}, \ldots, s_{4} \rightarrow i_{1}$

tentative matching: $i_{1}-s_{4}$

Phase 2:

offers: $s_{1} \rightarrow i_{4}$ and $s_{2}, s_{3} \rightarrow i_{2}$

tentative matching: $i_{1}-s_{4}, i_{2}-s_{2}$

Phase 3:

offers: $s_{1}, s_{3} \rightarrow i_{3}$

tentative matching: $i_{1}-s_{4}, i_{2}-s_{2}, i_{3}-s_{1}$

Phase 4:

offers: $s_{3} \rightarrow i_{4}$

final matching: $i_{1}-s_{4}, i_{2}-s_{2}, i_{3}-s_{1}, i_{4}-s_{3}$

CASE 2: $s_{2}$ is not acceptable in the truncation used by $i$, i.e., $P_{i}^{t}=\emptyset$ or $P_{i}^{t}=s_{1}$.

Phase 1:

offers: $s_{1}, \ldots, s_{4} \rightarrow i_{1}$

tentative matching: $i_{1}-s_{4}$

Phase 2:

offers: $s_{1} \rightarrow i_{4}$ and $s_{2}, s_{3} \rightarrow i_{2}$

tentative matching: $i_{1}-s_{4}$

Phase 3:

offers: $s_{1}, s_{3} \rightarrow i_{3}, s_{2} \rightarrow i_{4}$

tentative matching: $i_{1}-s_{4}, i_{3}-s_{1}, i_{4}-s_{2}$

Phase 4:

offers: $s_{3} \rightarrow i_{4}$

final matching: $i_{1}-s_{4}, i_{3}-s_{1}, i_{4}-s_{2}$

In each of the two cases, student $i_{2}$ is not matched to her most preferred school, which completes the proof of the claim. 


\section{Appendix B, additional figures and tables (intended for online publication)}
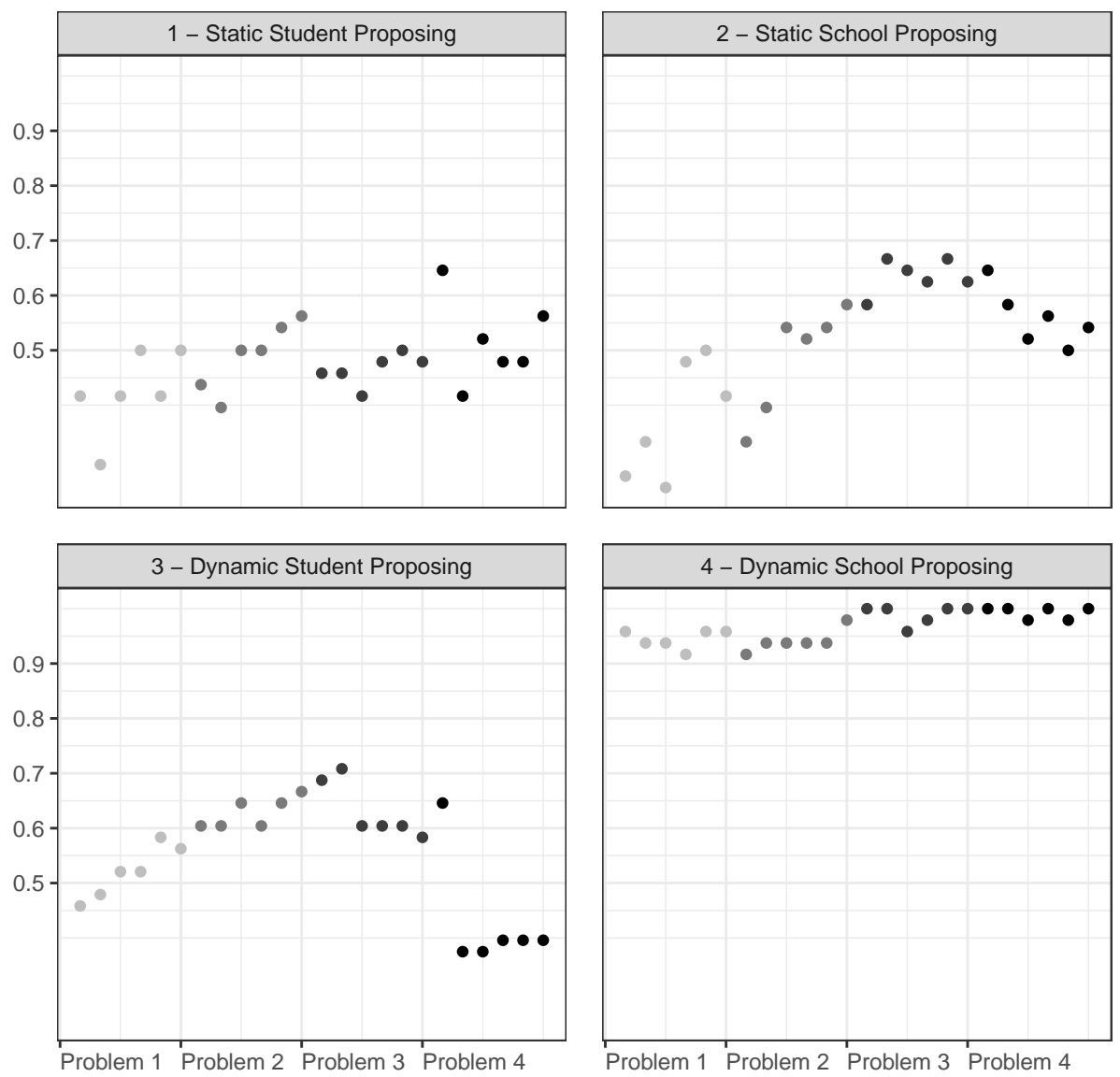

Figure 3: Dynamics that a subject cannot be rejected to tell the truth (student-proposing) and that a subject cannot be rejected to truncate (school-proposing). 

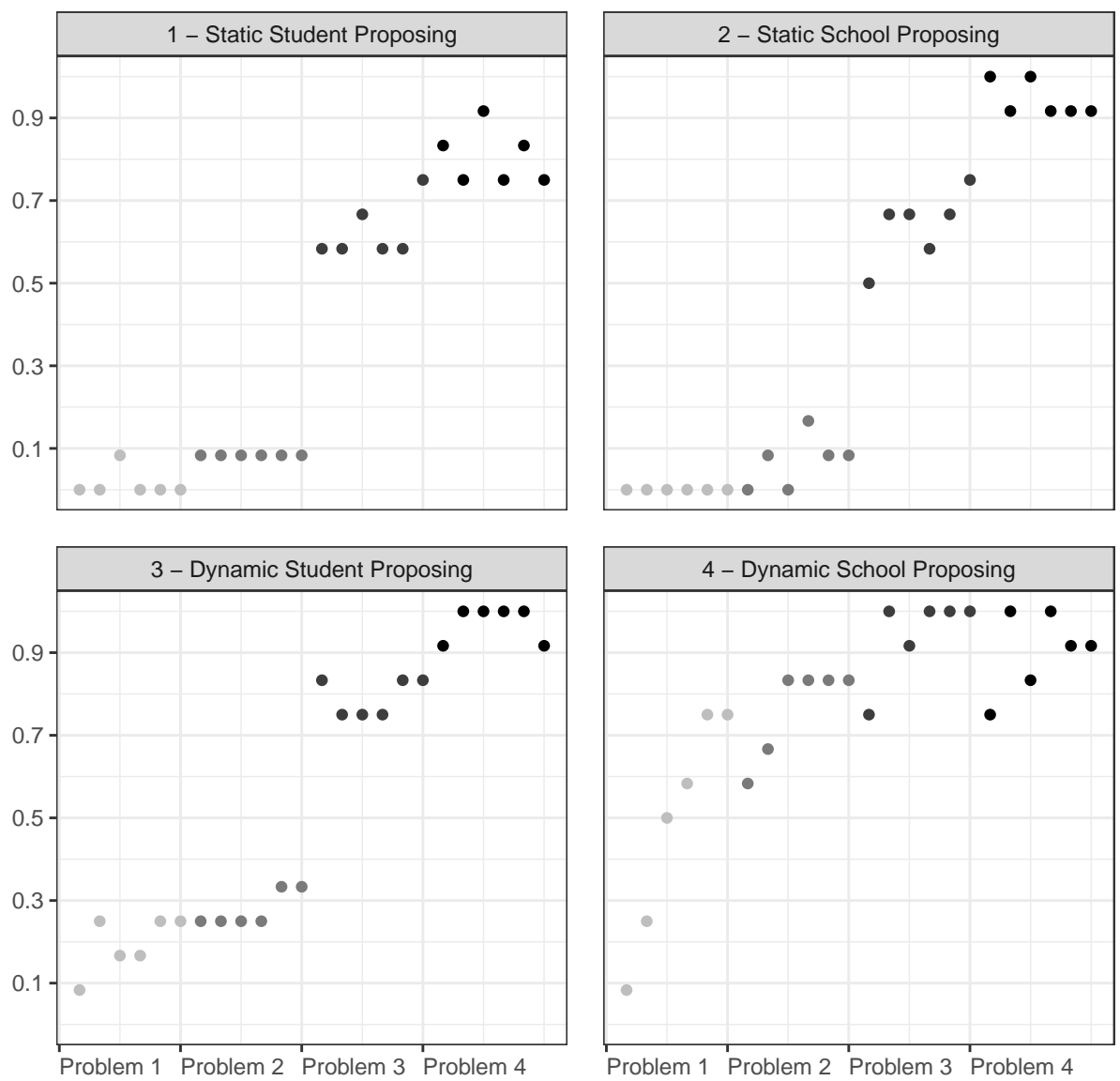

Figure 4: Frequency of the student-optimal stable matching. 


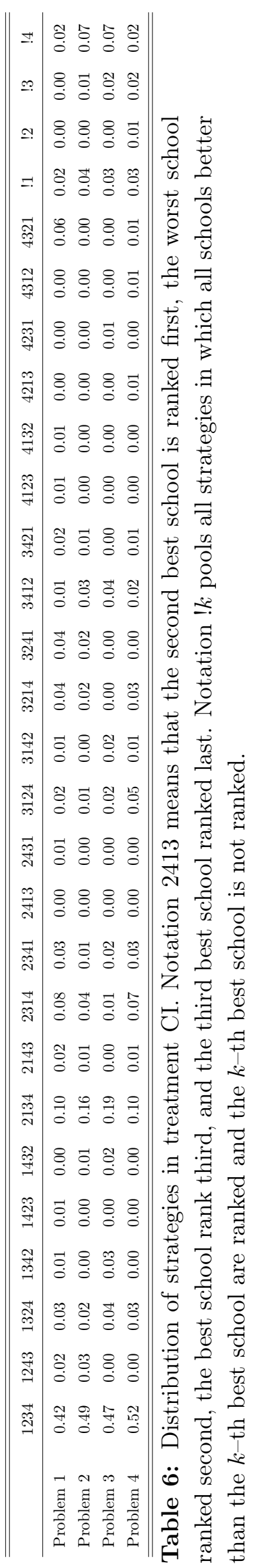




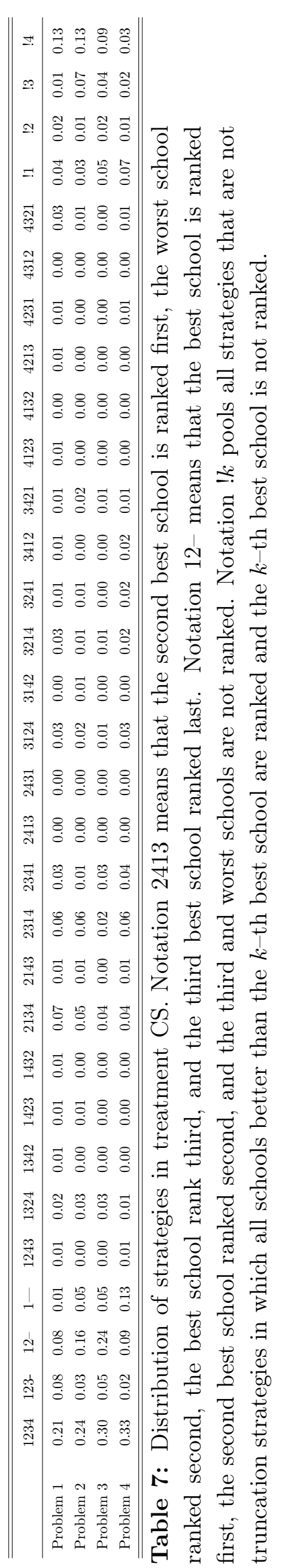

